\title{
Semi-Fredholm Solvability in the Framework of Singular Solutions for the (3+1)-D Protter-Morawetz Problem
}

\author{
Nedyu Popivanov, ${ }^{1}$ Todor Popov, ${ }^{1}$ and Allen Tesdall ${ }^{2}$ \\ ${ }^{1}$ Department of Mathematics and Informatics, University of Sofia, 1164 Sofia, Bulgaria \\ ${ }^{2}$ Department of Mathematics, City University of New York, College of Staten Island, Staten Island, NY 10314, USA
}

Correspondence should be addressed to Nedyu Popivanov; nedyu@yahoo.com

Received 28 February 2014; Accepted 11 May 2014; Published 14 October 2014

Academic Editor: Donal O’Regan

Copyright (c) 2014 Nedyu Popivanov et al. This is an open access article distributed under the Creative Commons Attribution License, which permits unrestricted use, distribution, and reproduction in any medium, provided the original work is properly cited.

For the four-dimensional nonhomogeneous wave equation boundary value problems that are multidimensional analogues of Darboux problems in the plane are studied. It is known that for smooth right-hand side functions the unique generalized solution may have a strong power-type singularity at only one point. This singularity is isolated at the vertex $O$ of the boundary light characteristic cone and does not propagate along the bicharacteristics. The present paper describes asymptotic expansions of the generalized solutions in negative powers of the distance to $O$. Some necessary and sufficient conditions for existence of bounded solutions are proven and additionally a priori estimates for the singular solutions are obtained.

\section{Introduction}

In the present paper, boundary value problems for the wave equation in $\mathbb{R}^{4}$

$$
u_{x_{1} x_{1}}+u_{x_{2} x_{2}}+u_{x_{3} x_{3}}-u_{t t}=f(x, t)
$$

with points $(x, t)=\left(x_{1}, x_{2}, x_{3}, t\right)$, are studied in the domain

$$
\Omega=\left\{(x, t): 0<t<\frac{1}{2}, t<\sqrt{x_{1}^{2}+x_{2}^{2}+x_{3}^{2}}<1-t\right\},
$$

bounded by the two characteristic cones

$$
\begin{gathered}
\Sigma_{1}=\left\{(x, t): 0<t<\frac{1}{2}, \sqrt{x_{1}^{2}+x_{2}^{2}+x_{3}^{2}}=1-t\right\}, \\
\Sigma_{2}=\left\{(x, t): 0<t<\frac{1}{2}, \sqrt{x_{1}^{2}+x_{2}^{2}+x_{3}^{2}}=t\right\},
\end{gathered}
$$

and the ball $\Sigma_{0}=\left\{t=0, \sqrt{x_{1}^{2}+x_{2}^{2}+x_{3}^{2}}<1\right\}$, centered at the origin $O: x=0, t=0$. The following BVPs were proposed by Protter [1].
Problem P2. Find a solution of the wave equation (1) in $\Omega$ which satisfies the boundary conditions

$$
P 2:\left.\quad u_{t}\right|_{\Sigma_{0}}=0,\left.\quad u\right|_{\Sigma_{1}}=0
$$

and its adjoint problem.

Problem $P 2^{*}$. Find a solution of the wave equation (1) in $\Omega$ which satisfies the adjoint boundary conditions

$$
P 2^{*}:\left.\quad u_{t}\right|_{\Sigma_{0}}=0,\left.\quad u\right|_{\Sigma_{2}}=0 .
$$

Protter [1] formulated in 1952 some versions of $P 2$ and $P 2^{*}$ in $\mathbb{R}^{3}$ (i.e., in (2+1)-D case) as a multidimensional analogue of the planar Darboux problems with boundary data prescribed on one characteristic and on the noncharacteristic segment. Initially the expectation was that such BVPs are classical solvable for very smooth right-hand side functions. However, soon it became clear that contrary to this traditional belief, unlike the plane Darboux problem, Protter's problems are not well posed. The reason is that the homogeneous adjoint Problem $P 2^{*}$ has an infinite number of nontrivial classical solutions (Tong [2], Popivanov and Schneider [3], and Khe [4]). It is known from [5] that for each $n \in \mathbb{N}$ there 
exists a right-hand side function $f \in C^{n}(\bar{\Omega})$ of the wave equation, for which the uniquely determined generalized solution of Problem $P 2$ has a strong power-type singularity like $r^{-n}$ at the origin $O$.

In the present paper we examine the exact behavior of the singular solutions of Problem P2. In the case when the righthand side function $f$ is harmonic polynomial, the Problem $P 2$ is Fredholm and we find the asymptotic expansion at $O$ of the unique generalized solution. On the other hand, in the general case when $f \in C^{1}(\bar{\Omega})$, the problem is not Fredholm because it has an infinite dimensional cokernel. We show that there are an infinite number of necessary conditions for the existence of bounded solutions. We discuss the semiFredholm solvability of Problem $P 2$ and for $f \in C^{6}(\bar{\Omega})$ we prove that the necessary conditions for the existence of bounded solutions are also sufficient.

In a historical perspective, Protter studied Problems $P 2$ and $P 2^{*}$ in connection with BVPs for mixed type equations that model transonic flow phenomena. In fact, in [1], he also proposes a multidimensional analogue to the twodimensional Guderley-Morawetz problem for the Gellerstedt equation of hyperbolic-elliptic type. The Guderley-Morawetz problem describes flows around airfoils and is well studied. The existence of weak solutions and the uniqueness of the strong ones were first established by Morawetz [6] by reducing the problem to a first-order system. Lax and Phillips [7] established that these weak solutions are strong. A survey for the classical 2D mixed-type BVPs and their transonic background can be found in [8]. The domain of Protter's analogue could be constructed by rotation in $\mathbb{R}^{4}$ of a symmetric planar domain for Guderley-Morawetz problem around the axis of symmetry. As a result the set $\Omega$ forms the hyperbolic part of the domain. Although it was expected that the multidimensional mixed-type problems would be similar to the two-dimensional BVPs, for the Protter hyperbolic-elliptic problems a general understanding of the situation is still not at hand. Even the question of well posedness is surprisingly subtle and not completely resolved. One has uniqueness results for quasiregular solutions, a class of solutions introduced by Protter, but there are real obstructions to existence in this class. The Protter problems in the hyperbolic part $\Omega$ of the domain illustrate some of the difficulties and differences between the planar BVPs and the multidimensional analogues.

In order to construct the solutions of the homogenous Problem $P 2^{*}$ we need the spherical functions $Y_{n}^{m}$ in $\mathbb{R}^{3}$. Traditionally, $Y_{n}^{m}$ are defined on the unit sphere $S^{2}$ := $\left\{\left(x_{1}, x_{2}, x_{3}\right): x_{1}^{2}+x_{2}^{2}+x_{3}^{2}=1\right\}$ (see [9]). For convenience in the discussions that follows, we keep the same notation $Y_{n}^{m}$ for the radial extension of the spherical function to $\mathbb{R}^{3} \backslash\{O\}$; that is, $Y_{n}^{m}(x):=Y_{n}^{m}(x /|x|)$ for $x \in \mathbb{R}^{3} \backslash\{O\}$. For the definition and properties of the spherical functions see Section 3. For $n, k \in \mathbb{N} \cup\{0\}$ define the functions

$$
E_{k}^{n}(x, t)=\sum_{i=0}^{k} B_{k, i}^{n} \frac{\left(|x|^{2}-t^{2}\right)^{n-k-i}}{|x|^{n-2 i+1}},
$$

where the coefficients are

$$
B_{k, i}^{n}:=(-1)^{i} \frac{(k-i+1)_{i}(n+1-k-i)_{i}}{i !(n-i+1 / 2)_{i}}, \quad B_{k, 0}^{n}=1,
$$

with $(a)_{i}:=a(a+1) \cdots(a+i-1)$ and $(a)_{0}:=1$. Then the functions

$$
W_{k, m}^{n}(x, t)=E_{k}^{n}(x, t) Y_{n}^{m}(x)
$$

are classical solutions of the homogeneous adjoint Protter problem.

Lemma 1 (see [10]). The functions $W_{k, m}^{n}(x, t)$ are classical solutions from $C(\bar{\Omega}) \cap C^{\infty}(\bar{\Omega} \backslash\{O\})$ of the homogeneous Problem $P 2^{*}$ for $k=0,1, \ldots,[n / 2]-1$.

A necessary condition for the existence of classical solution for the Problem $P 2$ is the orthogonality with respect to the $L^{2}(\Omega)$ inner product, of the right-hand side function $f$ to all functions $W_{k, m}^{n}(x, t)$ from Lemma 1 . To avoid an infinite number of necessary conditions in the framework of classical solvability, we introduce generalized solutions for the Problem $P 2$ (see the similar definition for the (2+1)-D case in [5]).

Definition 2 (see [10]). A function $u=u(x, t)$ is called a generalized solution of the Problem $P 2$ in $\Omega$, if the following conditions are satisfied:

(1) $u \in C^{1}(\bar{\Omega} \backslash O),\left.u_{t}\right|_{\Sigma_{0} \backslash O}=0,\left.u\right|_{\Sigma_{1}}=0$;

(2) the identity

$$
\int_{\Omega}\left(u_{t} w_{t}-u_{x_{1}} w_{x_{1}}-u_{x_{2}} w_{x_{2}}-u_{x_{3}} w_{x_{3}}-f w\right) d x d t=0
$$

holds for all $w \in C^{1}(\bar{\Omega})$ such that $\left.w_{t}\right|_{\Sigma_{0}}=0$ and $w=0$ in a neighborhood of $\Sigma_{2}$.

This definition allows the generalized solution to have singularity at the origin and there is a uniqueness result (see Theorem 18). Without any additional conditions imposed on the right-hand side function $f \in C(\bar{\Omega})$, it is known (see $[3,10])$ that the generalized solution may have power type singularity. Alternatively, we will prove the following necessary conditions for the existence of bounded solutions.

Theorem 3. Suppose that there is a bounded generalized solution of the Protter Problem P2 with right-hand side function $f(x, t) \in C(\bar{\Omega})$. Then

$$
\int_{\Omega} W_{k, m}^{n}(x, t) f(x, t) d x d t=0,
$$

for all $n \in \mathbb{N} \cup\{0\}, k=0, \ldots,[n / 2], m=1, \ldots, 2 n+1$.

The proof of Theorem 3 is given in Section 4, but before that we will describe the exact influence of the conditions (10) on the behavior of the generalized solution.

First, we consider the case when the right-hand side function $f \in C^{1}(\bar{\Omega})$ of the wave equation (1) has the representation

$$
f(x, t)=\sum_{n=0}^{l} \sum_{m=1}^{2 n+1} f_{n}^{m}(|x|, t) Y_{n}^{m}(x),
$$


with $l \in \mathbb{N} \cup\{0\}$. In particular, notice that in the case when $f_{n}^{m}(|x|, t)=|x|^{n} a_{n}^{m}(t)$ the function $f$ is a harmonic polynomial in $x$ of order $l$, whose coefficients are functions of $t$ (see the properties of $Y_{n}^{m}$ in Section 3). For convenience further by "harmonic polynomial of order $l$ " we will mean a function from $C^{1}(\bar{\Omega})$ that has the more general form (11). The coefficients $f_{n}^{m}$ are

$$
f_{n}^{m}(|x|, t)=\int_{S^{2}} f Y_{n}^{m} d \sigma
$$

and must have some special properties at $(0,0)$ (see, e.g., Lemma 17).

According to the results from [10] we know that the generalized solution of Problem $P 2$ may have a power type singularity at the origin $O: x=0, t=0$. In the present paper we study more accurately the exact behavior of the solution of Problem $P 2$ at $O$. It is governed by the parameters

$$
\beta_{k, m}^{n}:=\int_{\Omega} W_{k, m}^{n}(x, t) f(x, t) d x d t
$$

where $n=0, \ldots, l ; k=0, \ldots,[n / 2]$ and $m=1, \ldots, 2 n+1$. We find the asymptotic formula for the generalized solution of Problem $P 2$.

Theorem 4. Suppose that the right-hand side function $f \in$ $C^{1}(\bar{\Omega})$ has the form (11). Then the unique generalized solution $u(x, t)$ of Problem $P 2$ belongs to $C^{2}(\bar{\Omega} \backslash O)$ and has the following asymptotic expansion at the singular point $O$ :

$$
u(x, t)=\sum_{p=1}^{l+1}\left(|x|^{2}+t^{2}\right)^{-p / 2} F_{p}(x, t)+F(x, t),
$$

where

(i) the function $F \in C^{2}(\bar{\Omega} \backslash O)$ and satisfies the a priori estimate

$$
|F(x, t)| \leq C\|f\|_{C^{1}(\Omega)}, \quad(x, t) \in \Omega,
$$

with constant $C$ independent of $f$ and $\|f\|_{C^{k}(\Omega)}=$ $\sum_{|\alpha| \leq k} \max _{\bar{\Omega}}\left|D^{\alpha} f(x, t)\right| ;$

(ii) the functions $F_{p}, p=1, \ldots, l+1$, satisfy the equalities

$$
F_{p}(x, t)=\sum_{k=0}^{[(l-p+1) / 2]} \sum_{m=1}^{2 p+4 k-1} \beta_{k, m}^{p+2 k-1} F_{k, m}^{p+2 k-1}(x, t),
$$

with functions $F_{k, m}^{n} \in C^{2}(\bar{\Omega} \backslash O)$ bounded and independent of $f$;

(iii) if at least one of the constants $\beta_{k, m}^{p+2 k-1}$ in (16) is different from zero, then for the corresponding function $F_{p}(x, t)$ there exists a direction $(\alpha, 1):=\left(\alpha_{1}, \alpha_{2}, \alpha_{3}, 1\right)$ with $(\alpha, 1) t \in \Sigma_{2}$ for $0<t<1 / 2$, such that

$$
\lim _{t \rightarrow+0} F_{p}(\alpha t, t)=c_{p, \alpha}=\text { const } \neq 0 .
$$

After the case of the harmonic polynomials, here we deal with the more general situation when the right-hand side function $f$ is smooth, but it cannot be expanded simply as a sum (11). Now, Lemma 1 shows that the Problem $P 2$ is not Fredholm solvable.

Remark 5. Consider the operator

$$
T: \quad u_{f} \longmapsto f \in C^{k}(\bar{\Omega}),
$$

where $u_{f}$ is the unique classical solution to Protter Problem $P 2$ for the right-hand side function $f$. According to Lemma 1 we have $\operatorname{dim} \operatorname{coker}(T)=\infty$. This means that $T$ is not Fredholm operator for example in $C^{k}(\bar{\Omega})$. On the other hand, the uniqueness result Theorem 18 shows that $\operatorname{dim} \operatorname{ker}(T)=$ 0 and $T$ could be a semi-Fredholm operator. A semiFredholm operator is a bounded operator that has a finite dimensional kernel or cokernel and closed range (see, e.g., [11]). Accordingly we need to find the range of $T$.

The next result suggests that $T$ is a semi-Fredholm operator.

Theorem 6. Let the function $f(x, t)$ belong to $C^{6}(\bar{\Omega})$. Then the necessary and sufficient conditions for existence of bounded generalized solution $u(x, t)$ of the Protter Problem P2 are

$$
\int_{\Omega} W_{k, m}^{n}(x, t) f(x, t) d x d t=0
$$

for all $n \in \mathbb{N} \cup\{0\}, k=0, \ldots,[n / 2]$, and $m=1, \ldots, 2 n+1$.

Moreover, this generalized solution $u(x, t) \in C^{1}(\bar{\Omega} \backslash O)$ and satisfies the a priori estimates

$$
|u(x, t)| \leq C\left(\|f\|_{C^{5}(\bar{\Omega})}+\left\|f_{t}\right\|_{C^{5}(\bar{\Omega})}\right) ;
$$

$$
\sum_{i=1}^{3}\left|u_{x_{i}}(x, t)\right|+\left|u_{t}(x, t)\right| \leq C\left(|x|^{2}+t^{2}\right)^{-1}\|f\|_{C^{6}(\bar{\Omega})},
$$

where the constant $C$ is independent of the function $f(x, t)$.

Obviously, the set of all functions from $C^{6}(\bar{\Omega})$ that satisfy the orthogonality conditions (19) is closed. Therefore, Theorem 6 shows that the operator $T$ defined in Remark 5 with a domain $D(T) \subset C(\bar{\Omega})$ has a closed range in $C^{6}(\bar{\Omega})$, and we get the following result.

Corollary 7. The operator $T$ is a semi-Fredholm operator from $D(T) \subset C(\bar{\Omega})$ to $C^{6}(\bar{\Omega})$.

We have briefly announced some of the results from this section in [12] with the assumption $f \in C^{9}(\bar{\Omega})$.

The main results in this work are discussed in Section 2 and the proofs are in Sections 3-7. In more detail the paper is organized as follows: estimates for the spherical functions involved in the representation of the solution are proven in Section 3. In Section 4 the necessary conditions for bounded solution Theorem 3 are proved. In Section 5 we consider some two-dimensional boundary value problems connected 
to Problem $P 2$, the Problems $P 21$ and $P 22$. Exact formulas for the solution of the Problem $P 22$ are presented in Lemma 20. In Section 6 the proofs of the main Theorems 4 and 6 are given based on the results from the previous sections and an asymptotic expansion formula for the generalized solution of the 2D Problem P21 (Theorem 22). The long and technical proof of Theorem 22 is postponed to Section 7.

\section{Historical Remarks on the Main Results}

Let us point out several related recent works on Protter problems. Necessary and sufficient conditions for the existence of solutions with fixed order of singularity were obtained in [10]. Similarly, for the $\mathbb{R}^{3}$-analogues of Protter problems, some results are presented in $[13,14]$. For the problem with Dirichlet type boundary condition on $\Sigma_{0}$, a formula for the asymptotic expansion of the singular solution can be found in [15], and the semi-Fredholm solvability is discussed in [16] for $f \in C^{10}(\bar{\Omega})$. A comparison of various recent results for Protter problems is made in [13].

Various authors adopted a variety of approaches to Protter problems over the last sixty years, for example, WienerHopf method, special Legendre functions, a priori estimates, nonlocal regularization, and so forth (see [5] and references therein; see also [4, 10, 14, 17-19]). Alternatively, different multidimensional analogues of the classical Darboux problem for the wave equation are considered in [20-22], while for some related semilinear equations and systems see [23]. The existence of bounded or unbounded solutions for the wave equation in $\mathbb{R}^{3}$ and $\mathbb{R}^{4}$, as well as for the Euler-PoissonDarboux equation, has been studied in $[4,10,17,19,24-26]$.

Regarding the Protter problems with lower order terms see [27] and references therein. Problems with more general boundary condition $u_{t}+\alpha(x) u=0$ on $\Sigma_{0}$ are studied in $[26,27]$. Some possible regularization methods involving integrodifferential or nonlocal terms can be found in [18].

For the Protter problems for equations of mixed hyperbolic-elliptic type proposed in [1], Aziz and Schneider [28] proved an uniqueness result in the linear case (see also [21]). Concerning nonexistence principle for nontrivial solution of semilinear mixed-type equations in multidimensional case, we refer to [29].

In 1960 Garabedian [30] proved the uniqueness of a classical solution of Proter problem. However, generally, Problem $P 2$ is not classically solvable and a necessary condition for the existence of a classical solution is the orthogonality of the right-hand side function $f$ to all solutions of the corresponding homogeneous adjoint Problem $P 2^{*}$. Here, in Lemma 1, the solutions $W_{k, m}^{n}$ were constructed with the help of the functions $E_{k}^{n}$ defined by (6). The alternate representation in terms of the Gauss hypergeometric function $F=F(a, b, c ; x)$

$$
\begin{aligned}
E_{k}^{n}(x, t)= & |x|^{n-2 k-1}\left(1-\frac{t^{2}}{|x|^{2}}\right)^{n-2 k} \\
& \times F\left(n-k+\frac{1}{2},-k, \frac{1}{2} ; \frac{t^{2}}{|x|^{2}}\right)
\end{aligned}
$$

can be found in Khe [4]. In [3] there are some solutions for the three-dimensional analogue of the homogeneous Problem $P 2^{*}$.

Let us look back at Theorem 3 and the necessary orthogonality conditions (10) for the existence of bounded solutions of Problem P2. Naturally, these conditions include the functions $W_{k, m}^{n}$ from Lemma 1 . However, notice that there are also some others.

Remark 8. It is interesting that conditions (10) include the case of even $n=2 k$. Notice that the functions $W_{k, m}^{2 k}(x, t)$ are not classical solutions of the homogenous adjoint Problem $P 2^{*}$. Actually, they satisfy the homogenous wave equation in $\Omega$ and $\left(W_{k, m}^{2 k}\right)_{t}$ vanish on $\Sigma_{0}$, but $W_{k, m}^{2 k}$ is not zero on $\Sigma_{2}$. In addition, the functions $W_{k, m}^{2 k}$ have a singularity at the origin $O$ like $|x|^{-1}$; however, this singularity is integrable in the domain $\Omega$.

Instead of imposing an infinite number of orthogonality conditions on $f$, Popivanov and Schneider $[3,5]$ introduced the concept of generalized solution that allows the solution to have singularity on the inner cone $\Sigma_{2}$. Here, Theorem 4 describes the effect of the parameters $\beta_{k, m}^{n}$ on the behavior of the generalized solution of Problem P2. The constants $\beta_{k, m}^{n}$ are defined by (13) and are obviously related to the orthogonality conditions (10). When the right-hand function is a harmonic polynomial (11), the asymptotic expansion in Theorem 4 shows that the generalized solution could be bounded only if all $\beta_{k, m}^{n}$ involved are zero.

Corollary 9. Suppose that the right-hand side function $f \in$ $C^{1}(\bar{\Omega})$ has the form (11) and satisfies the orthogonality conditions

$$
\int_{\Omega} W_{k, m}^{n}(x, t) f(x, t) d x d t=0,
$$

for all $n=0, \ldots, l ; k=0, \ldots,[n / 2]$ and $m=1, \ldots, 2 n+1$. Then the unique generalized solution $u(x, t)$ of Problem P2 belongs to $C^{2}(\bar{\Omega} \backslash O)$, is bounded, and satisfies the a priori estimate

$$
\max _{\bar{\Omega}}|u| \leq C\|f\|_{C^{1}(\Omega)} .
$$

On the other hand, without any orthogonality conditions on $f$, the following result is obtained.

Corollary 10. The generalized solution $u$ of Problem P2 with a right-hand side function $f \in C^{1}(\bar{\Omega})$ in the form (11) satisfies the a priori estimate

$$
|u(x, t)| \leq C\left(\max _{\bar{\Omega}}|f|\right)\left(|x|^{2}+t^{2}\right)^{-(l+1) / 2} .
$$

The influence of the orthogonality conditions (22) on the exact behavior of the generalized solution is clarified by case (iii) of Theorem 4. It shows that, for fixed indexes $(n, k, m)$, the corresponding condition (22) "controls" one power-type singularity.

In Corollaries 9 and 10 the emphasis is on the extreme cases: when all orthogonality conditions (22) are fulfilled or, alternatively, when none of them are satisfied. In both 
cases the exact behavior of the solution is given. The estimate (24), presented here, is analogous to known estimates for Protter problems in $\mathbb{R}^{3}[5]$ and in $\mathbb{R}^{m}$ [24]. It is interesting that singularities of the generalized solutions are isolated at the origin and do not propagate in the direction of the bicharacteristics on the characteristic cone $\Sigma_{2}$. Traditionally, it is assumed that the wave equation, with sufficiently smooth right-hand side, cannot have a solution with an isolated singular point as in Hörmander [31, Chapter 24.5]. The case here is different since the point of singularity $O$ lies on the noncharacteristic part of the boundary $\Sigma_{0}$, as well as on the characteristic part $\Sigma_{2}$.

Remark 11. The Problem $P 2$ in $\mathbb{R}^{4}$ with harmonic polynomial on the right-hand side is also studied in [10]. However, the explicit asymptotic expansion here has no analogue in [10], where only the behavior of the singularities is given. Additionally, if the orthogonality conditions (22) are fulfilled, Corollary 9 states that the generalized solution is in fact bounded, while the estimates in [10, Theorem 1.1] still allow the solution to have some logarithmic singularities.

Remark 12. Let us compare Protter problems in $\mathbb{R}^{3}$ (as treated in $[13,14]$ ) and $\mathbb{R}^{4}$ here (see also $[10,15]$ ). In both cases the study of these BVPs is based on the properties of the special Legendre functions. Instead of Legendre polynomials $P_{n}$ here, in the three-dimensional case, the Legendre functions $P_{v}$ with noninteger indexes $v=n-1 / 2$ are used (for their properties see [32]). One can easily modify both these techniques to obtain similar results for the $(m+1)$-dimensional problems, for even $m$ (analogous to $\mathbb{R}^{3}$ case) or for odd $m$ (the present case $\mathbb{R}^{4}$ ). Some related results for Protter problems in $\mathbb{R}^{m+1}$ are presented in $[17,24]$.

In the general case when the right-hand side function $f$ is smooth enough, Theorem 6 implies that the necessary conditions (10) for existence of bounded solutions from Theorem 3 are also sufficient. Further, this means that there are no other nontrivial classical solutions of the homogenous adjoint Problem P2* except those listed in Lemma 1.

Remark 13. We point out the differences between Theorem 6 and the results from [16] for Protter Problem $P 1$ with Dirichlet type boundary condition on $\Sigma_{0}$. First, notice that in the case when right-hand side function $f$ is harmonic polynomial of order $l$ the solution of Problem $P 2$ may have worse singularity (like $\left(|x|^{2}+t^{2}\right)^{-(l+1) / 2}$; see Theorem 4) than the solution of Problem $P 1$ (like $\left(|x|^{2}+t^{2}\right)^{-l / 2}$; see [15]). For the general case we are able to reduce the assumptions on $f$; in Theorem 6 we assume $f \in C^{6}(\bar{\Omega})$, while in [16, Theorem 1.1] smoother $f \in C^{10}(\bar{\Omega})$ is required. In order to achieve this, we rely on the more accurate estimates for the special functions proven in Section 3.

\section{Estimates for the Special Functions}

For the proof of the main results we will need some properties of the spherical functions $Y_{n}^{m}$ in $\mathbb{R}^{3}$. They are naturally expressed on the unit sphere $S^{2}:=\left\{\left(x_{1}, x_{2}, x_{3}\right): x_{1}^{2}+x_{2}^{2}+\right.$ $\left.x_{3}^{2}=1\right\}$ in spherical polar coordinates. Let us introduce polar coordinates $(r, \theta, \varphi)$ in $\mathbb{R}^{3}$ :

$$
x_{1}=r \sin \theta \cos \varphi, \quad x_{2}=r \sin \theta \sin \varphi, \quad x_{3}=r \cos \theta,
$$

where $0 \leq \theta<\pi, 0 \leq \varphi<2 \pi, r>0$. Then the spherical functions, expressed in terms of $\theta$ and $\varphi$ as in the traditional definition on $S^{2}$ (see [9]), are given by

$$
\begin{array}{r}
Y_{n}^{2 k+1}(\theta, \varphi)=\sqrt{\frac{2 n+1}{2 \pi}} \sqrt{\frac{(n-k) !}{(n+k) !}} P_{n}^{k}(\cos \theta) \cos k \varphi, \\
k=1, \ldots, n, \\
Y_{n}^{2 k}(\theta, \varphi)=\sqrt{\frac{2 n+1}{2 \pi}} \sqrt{\frac{(n-k) !}{(n+k) !}} P_{n}^{k}(\cos \theta) \sin k \varphi, \\
k=1, \ldots, n,
\end{array}
$$

and $Y_{n}^{1}(\theta, \varphi)=((2 n+1) / 4 \pi)^{1 / 2} P_{n}(\cos \theta)$. Here $P_{n}$ are the Legendre polynomials defined by the Rodrigues' formula

$$
P_{n}(s):=\frac{1}{2^{n} n !} \frac{d^{n}}{d s^{n}}\left(s^{2}-1\right)^{n}=\sum_{k=0}^{[n / 2]} a_{2 k} s^{n-2 k}, \quad a_{2 k} \neq 0,
$$

while $P_{n}^{k}$ are the associated Legendre polynomials that can be defined as

$$
P_{n}^{k}(s)=(-1)^{k}\left(1-s^{2}\right)^{k / 2} \frac{d^{k}}{d s^{k}} P_{n}(s) .
$$

The functions $Y_{n}^{m}$ satisfy the differential equation

$$
\frac{1}{\sin \theta} \frac{\partial}{\partial \theta}\left(\sin \theta \frac{\partial}{\partial \theta} Y_{n}^{m}\right)+\frac{1}{\sin ^{2} \theta} \frac{\partial^{2}}{\partial \varphi^{2}} Y_{n}^{m}+n(n+1) Y_{n}^{m}=0
$$

and form a complete orthonormal system in $L_{2}\left(S^{2}\right)$ (see [9]).

Using Cartesian coordinates as in Section 1, one can define the spherical functions as $Y_{n}^{m}\left(x_{1}, x_{2}, x_{3}\right):=Y_{n}^{m}(\theta, \varphi)$ for $x \in S^{2}$, or by

$$
\begin{array}{r}
Y_{n}^{2 m}\left(x_{1}, x_{2}, x_{3}\right)=C_{n}^{m} \frac{d^{m}}{d x_{3}^{m}} P_{n}\left(x_{3}\right) \operatorname{Im}\left\{\left(x_{2}+i x_{1}\right)^{m}\right\}, \\
\text { for } m=1, \ldots, n, \\
Y_{n}^{2 m+1}\left(x_{1}, x_{2}, x_{3}\right)=C_{n}^{m} \frac{d^{m}}{d x_{3}^{m}} P_{n}\left(x_{3}\right) \operatorname{Re}\left\{\left(x_{2}+i x_{1}\right)^{m}\right\}, \\
\text { for } m=0, \ldots, n,
\end{array}
$$

where $C_{n}^{m}$ are constants. In the present paper, we keep the same notation $Y_{n}^{m}$ for the radial extension of the spherical function to $\mathbb{R}^{3} \backslash\{O\}$; that is, $Y_{n}^{m}(x):=Y_{n}^{m}(x /|x|)$ for $x \in$ $\mathbb{R}^{3} \backslash\{O\}$. According to the properties of $Y_{n}^{m}(x)$, the function 
$|x|^{n} Y_{n}^{m}(x)$ is a homogenous harmonic polynomial of order $n$ in the variables $x_{1}, x_{2}, x_{3}$.

We will need some estimates for $Y_{n}^{m}$ and the special functions involved in the representations of the solutions of the Protter problems. Let us start with the Legendre polynomials $P_{n}$.

Lemma 14. The following estimates hold for $x \in[0,1]$ :

$$
\begin{gathered}
\left|P_{n}(x)\right| \leq 1, \quad\left|P_{n}^{\prime}(x)\right| \leq \frac{n(n+1)}{2}, \\
\left|P_{n}(x)-P_{n}(0)\right| \leq n x .
\end{gathered}
$$

Proof. The estimates (31) are proved, for example, in [10]. Here we will show that (32) holds. Using Bonnet's recursion formula

$$
(n+1) P_{n+1}(x)=(2 n+1) x P_{n}(x)-n P_{n-1}(x),
$$

we get $(n+1) P_{n+1}(0)=-n P_{n-1}(0)$ and

$$
\begin{aligned}
(n+1) & \left(P_{n+1}(x)-P_{n+1}(0)\right) \\
= & (2 n+1) x P_{n}(x)-n\left(P_{n-1}(x)-P_{n-1}(0)\right) .
\end{aligned}
$$

Thus

$$
\left|P_{n+1}(x)-P_{n+1}(0)\right| \leq 2 x+\left|P_{n-1}(x)-P_{n-1}(0)\right| \text {. }
$$

From here and the equalities $P_{0}(x)-P_{0}(0)=0$ and $P_{1}(x)-$ $P_{1}(0)=x$ we get the estimate $(32)$ by induction.

In order to study the first derivatives of the generalized solution of the Protter problem, we will need to estimate also the first derivatives with respect to $x$ of $Y_{n}^{m}(x):=Y_{n}^{m}(x /|x|)$, the radial extension of the spherical function to $\mathbb{R}^{3} \backslash\{O\}$ :

$$
\begin{gathered}
\frac{\partial}{\partial x_{1}} Y_{n}^{m}:=r^{-1}\left[\cos \theta \cos \varphi\left(Y_{n}^{m}\right)_{\theta}-(\sin \theta)^{-1} \sin \varphi\left(Y_{n}^{m}\right)_{\varphi}\right] \\
\frac{\partial}{\partial x_{2}} Y_{n}^{m}=r^{-1}\left[\cos \theta \sin \varphi\left(Y_{n}^{m}\right)_{\theta}+(\sin \theta)^{-1} \cos \varphi\left(Y_{n}^{m}\right)_{\varphi}\right] \\
\frac{\partial}{\partial x_{3}} Y_{n}^{m}:=-r^{-1} \sin \theta\left(Y_{n}^{m}\right)_{\theta}
\end{gathered}
$$

Using the so-called addition theorem for Legendre polynomials we get the following result.

Lemma 15. For $n \geq 1$ the functions $Y_{n}^{m}(x)$ satisfy the equalities

$$
\begin{gathered}
\sum_{m=1}^{2 n+1}\left(Y_{n}^{m}\right)^{2}=\frac{2 n+1}{4 \pi} \\
\sum_{m=1}^{2 n+1} \sum_{i=1}^{3}\left(\frac{\partial}{\partial x_{i}} Y_{n}^{m}\right)^{2}=\frac{n(n+1)(2 n+1)}{2 \pi|x|^{2}} .
\end{gathered}
$$

Proof. From the definition (26) of $Y_{n}^{m}$ it follows that

$$
\begin{aligned}
\sum_{m=1}^{2 n+1}\left(Y_{n}^{m}\right)^{2}=\frac{2 n+1}{4 \pi}[ & \left(P_{n}(\cos \theta)\right)^{2} \\
& \left.+2 \sum_{k=1}^{n} \frac{(n-k) !}{(n+k) !}\left(P_{n}^{k}(\cos \theta)\right)^{2}\right] .
\end{aligned}
$$

According to the addition theorem (see [33])

$$
\begin{aligned}
& P_{n}\left(\cos \theta \cos \theta_{1}+\sin \theta \sin \theta_{1} \cos \varphi\right) \\
& =P_{n}(\cos \theta) P_{n}\left(\cos \theta_{1}\right) \\
& \quad+2 \sum_{k=1}^{n} \frac{(n-k) !}{(n+k) !} P_{n}^{k}(\cos \theta) P_{n}^{k}\left(\cos \theta_{1}\right) \cos k \varphi .
\end{aligned}
$$

With $\theta=\theta_{1}$ and $\varphi=0$ one derives the equality

$$
\left(P_{n}(\cos \theta)\right)^{2}+2 \sum_{k=1}^{n} \frac{(n-k) !}{(n+k) !}\left(P_{n}^{k}(\cos \theta)\right)^{2}=1 .
$$

This means that

$$
\sum_{m=1}^{2 n+1}\left(Y_{n}^{m}\right)^{2}=\frac{2 n+1}{4 \pi} .
$$

Using (39) again, we get the required property of the derivatives of $Y_{n}^{m}$. Directly from (36) for the squares of the derivatives with respect to $x$ we have

$$
\sum_{|\alpha|=1}\left(D_{x}^{\alpha} Y_{n}^{m}\right)^{2}=r^{-2}\left(Y_{n}^{m}\right)_{\theta}^{2}+r^{-2}(\sin \theta)^{-2}\left(Y_{n}^{m}\right)_{\varphi}^{2},
$$

and from the definition (26) of $Y_{n}^{m}$ we find

$$
\begin{aligned}
& \sum_{m=1}^{2 n+1}\left(\frac{\partial Y_{n}^{m}}{\partial \theta}\right)^{2}=\frac{2 n+1}{4 \pi}[\left(\frac{\partial}{\partial \theta} P_{n}(\cos \theta)\right)^{2} \\
&\left.+2 \sum_{k=1}^{n} \frac{(n-k) !}{(n+k) !}\left(\frac{\partial}{\partial \theta} P_{n}^{k}(\cos \theta)\right)^{2}\right] \\
& \sum_{m=1}^{2 n+1}\left(\frac{\partial Y_{n}^{m}}{\partial \varphi}\right)^{2}=\frac{2 n+1}{2 \pi} \sum_{k=1}^{n} k^{2} \frac{(n-k) !}{(n+k) !}\left(P_{n}^{k}(\cos \theta)\right)^{2}
\end{aligned}
$$

Put $\varphi=0$, and differentiation of (39) with respect to $\theta$ and $\theta_{1}$ gives

$$
\begin{aligned}
P_{n}^{\prime \prime}(\cos \theta & \left.\theta \cos \theta_{1}+\sin \theta \sin \theta_{1}\right)\left\{\cos \theta \sin \theta_{1}-\sin \theta \cos \theta_{1}\right\} \\
& \times\left\{\sin \theta \cos \theta_{1}-\cos \theta \sin \theta_{1}\right\} \\
& +P_{n}^{\prime}\left(\cos \theta \cos \theta_{1}+\sin \theta \sin \theta_{1}\right) \\
& \times\left\{\cos \theta \cos \theta_{1}+\sin \theta \sin \theta_{1}\right\} \\
= & \sin \theta \sin \theta_{1} P_{n}^{\prime}(\cos \theta) P_{n}^{\prime}\left(\cos \theta_{1}\right)+2 \sin \theta \sin \theta_{1} \\
& \times \sum_{k=1}^{n} \frac{(n-k) !}{(n+k) !}\left(P_{n}^{k}\right)^{\prime}(\cos \theta)\left(P_{n}^{k}\right)^{\prime}\left(\cos \theta_{1}\right) .
\end{aligned}
$$


With $\theta=\theta_{1}$ we derive

$$
\left(\frac{\partial}{\partial \theta} P_{n}(\cos \theta)\right)^{2}+2 \sum_{k=1}^{n} \frac{(n-k) !}{(n+k) !}\left(\frac{\partial}{\partial \theta} P_{n}^{k}(\cos \theta)\right)^{2}=P_{n}^{\prime}(1) .
$$

Analogously, after differentiating (39) twice with respect to $\varphi$,

$$
\begin{aligned}
P_{n}^{\prime \prime}\left(\cos \theta \cos \theta_{1}+\sin \theta \sin \theta_{1} \cos \varphi\right) \sin ^{2} \theta \sin ^{2} \theta_{1} \sin ^{2} \varphi \\
\quad-P_{n}^{\prime}\left(\cos \theta \cos \theta_{1}+\sin \theta \sin \theta_{1} \cos \varphi\right) \sin \theta \sin \theta_{1} \cos \varphi \\
=-2 \sum_{k=1}^{n} k^{2} \frac{(n-k) !}{(n+k) !} P_{n}^{k}(\cos \theta) P_{n}^{k}\left(\cos \theta_{1}\right) \cos k \varphi .
\end{aligned}
$$

Then substituting $\varphi=0$ and $\theta_{1}=\theta$ we have

$$
2 \sum_{k=1}^{n} k^{2} \frac{(n-k) !}{(n+k) !}\left(P_{n}^{k}(\cos \theta)\right)^{2}=(\sin \theta)^{2} P_{n}^{\prime}(1) .
$$

Since $P_{n}^{\prime}(1)=n(n+1) / 2$ we conclude that

$$
\sum_{m=1}^{2 n+1} \sum_{|\alpha|=1}\left(D_{x}^{\alpha} Y_{n}^{m}\right)^{2}=\frac{n(n+1)(2 n+1)}{2 \pi r^{2}}
$$

As a direct consequence, we find the next estimates for the radial extension of the spherical functions to $\mathbb{R}^{3} \backslash\{O\}$.

Corollary 16. For $n \geq 1$ the functions $Y_{n}^{m}(x)$ satisfy the inequalities

$$
\begin{gathered}
\left|Y_{n}^{m}(x)\right| \leq n^{1 / 2}, \\
\sum_{m=1}^{2 n+1}\left|Y_{n}^{m}(x)\right| \leq n, \\
\sum_{m=1}^{2 n+1} \sum_{i=1}^{3}\left|\frac{\partial}{\partial x_{i}} Y_{n}^{m}(x)\right| \leq 3 n^{2}|x|^{-1}, \\
\sum_{m=1}^{2 n+1}\left[\left|\left(Y_{n}^{m}\right)_{\theta}\right|+\left|(\sin \theta)^{-1}\left(Y_{n}^{m}\right)_{\varphi}\right|\right] \leq 3 n^{2} .
\end{gathered}
$$

Proof. Obviously

$$
\left|Y_{n}^{m}(x)\right| \leq\left[\sum_{m=1}^{2 n+1}\left(Y_{n}^{m}\right)^{2}\right]^{1 / 2} \leq n^{1 / 2} .
$$

The estimates (50) and (51) follow directly from the CauchySchwarz inequality and Lemma 15:

$$
\begin{aligned}
\sum_{m=1}^{2 n+1}\left|Y_{n}^{m}(x)\right| & \leq \sqrt{2 n+1}\left[\sum_{m=1}^{2 n+1}\left(Y_{n}^{m}\right)^{2}\right]^{1 / 2} \\
& =\frac{2 n+1}{2 \sqrt{\pi}} \leq n
\end{aligned}
$$

$$
\begin{aligned}
\sum_{m=1}^{2 n+1} \sum_{i=1}^{3} & \left|\frac{\partial}{\partial x_{i}} Y_{n}^{m}(x)\right| \\
& \leq \sqrt{6 n+3}\left[\sum_{m=1}^{2 n+1} \sum_{i=1}^{3}\left(\frac{\partial}{\partial x_{i}} Y_{n}^{m}\right)^{2}\right]^{1 / 2} \\
& =\frac{(2 n+1) \sqrt{3 n(n+1)}}{\sqrt{2 \pi}|x|} .
\end{aligned}
$$

Finally (52) follows from the equality (42):

$$
\begin{aligned}
& \sum_{m=1}^{2 n+1}\left\{\left|\left(Y_{n}^{m}\right)_{\theta}\right|+\left|(\sin \theta)^{-1}\left(Y_{n}^{m}\right)_{\varphi}\right|\right\} \\
& \leq \sqrt{4 n+2}\left\{\sum_{m=1}^{2 n+1}\left[\left(Y_{n}^{m}\right)_{\theta}^{2}+(\sin \theta)^{-2}\left(Y_{n}^{m}\right)_{\varphi}^{2}\right]\right\}^{1 / 2} \\
& \quad=|x| \sqrt{4 n+2}\left[\sum_{m=1}^{2 n+1} \sum_{i=1}^{3}\left(\frac{\partial}{\partial x_{i}} Y_{n}^{m}\right)^{2}\right]^{1 / 2} \leq 3 n^{2} .
\end{aligned}
$$

Generally, if the function $f(x, t)$ is smooth enough, it can be represented as a harmonic series. In order to estimate the coefficients of the series we will use the next result. It is based on the fact that the spherical functions are eigenfunctions for the Laplace operator on the sphere $S^{2}$.

Lemma 17. Let $k \in \mathbb{N} \cup\{0\}$.

(1) (See [16]). Let $f \in C^{2 k}(\bar{\Omega})$. Then it has the representation

$$
f(x, t)=\sum_{n=0}^{\infty} \sum_{m=1}^{2 n+1} f_{n}^{m}(|x|, t) Y_{n}^{m}(x),
$$

and for $n \in \mathbb{N}$

$$
\left|f_{n}^{m}\right| \leq C n^{-2 k}\|f\|_{C^{2 k}(\bar{\Omega})} .
$$

(2) Suppose $f \in C^{2 k+1}(\bar{\Omega})$; then for $n \in \mathbb{N}$

$$
\sum_{m=1}^{2 n+1}\left|f_{n}^{m}\right| \leq C n^{-2 k}|x|\|f\|_{C^{2 k+1}(\bar{\Omega})} .
$$

Proof. The estimate in case (1) follows directly from the fact that the spherical functions are eigenfunctions for the spherical Laplacean $\Delta_{S}$ :

$$
\Delta_{S} F:=\frac{1}{\sin \theta} \frac{\partial}{\partial \theta}\left(\sin \theta \frac{\partial}{\partial \theta} F\right)+\frac{1}{\sin ^{2} \theta} \frac{\partial^{2}}{\partial \varphi^{2}} F .
$$

That is, (29) shows that $\Delta_{S} Y_{n}^{m}=-n(n+1) Y_{n}^{m}$. In fact $f_{n}^{m}$ is bounded by the Fourier coefficient of $\left(\Delta_{S}\right)^{k} f$ :

$$
\left|f_{n}^{m}\right|=\left|\int_{S^{2}} Y_{n}^{m} f d \sigma\right| \leq C n^{-2 k}\left|\int_{S^{2}} Y_{n}^{m}\left(\Delta_{S}\right)^{k} f d \sigma\right| .
$$

For detail proof of (1) see [16, 34]. 

then

Next we will prove case (2) with $k=0$. Let $F \in C^{1}(\bar{\Omega})$;

$$
\begin{aligned}
F_{n}^{m}(r, t):= & \int_{S^{2}} Y_{n}^{m} F d \sigma \\
= & -n^{-1}(n+1)^{-1} \\
& \times \int_{0}^{\pi}\left(\int_{0}^{2 \pi}\left(\Delta_{S} Y_{n}^{m}\right) F d \varphi\right) \sin \theta d \theta .
\end{aligned}
$$

Integrating by parts gives

$$
\begin{aligned}
F_{n}^{m}=\frac{1}{n(n+1)} \int_{0}^{\pi}\left[\int_{0}^{2 \pi}(\right. & \sin \theta \frac{\partial}{\partial \theta} Y_{n}^{m} \frac{\partial}{\partial \theta} F \\
& \left.\left.+\frac{1}{\sin \theta} \frac{\partial}{\partial \varphi} Y_{n}^{m} \frac{\partial}{\partial \varphi} F\right) d \varphi\right] d \theta
\end{aligned}
$$

where

$$
\begin{aligned}
\sin \theta \frac{\partial}{\partial \theta} F= & r \sin \theta \cos \theta \cos \varphi \frac{\partial}{\partial x_{1}} F \\
& +r \sin \theta \cos \theta \sin \varphi \frac{\partial}{\partial x_{2}} F-r \sin ^{2} \theta \frac{\partial}{\partial x_{3}} F \\
\frac{\partial}{\partial \varphi} F= & -r \sin \theta \sin \varphi \frac{\partial}{\partial x_{1}} F+r \sin \theta \cos \varphi \frac{\partial}{\partial x_{2}} F
\end{aligned}
$$

Therefore using (52) we have

$$
\begin{aligned}
& \sum_{m=1}^{2 n+1}\left|F_{n}^{m}\right| \\
& \leq \frac{C|x|}{n^{2}}\|F\|_{C^{1}(\bar{\Omega})} \sum_{m=1}^{2 n+1} \int_{0}^{\pi}\left[\int _ { 0 } ^ { 2 \pi } \left(\left|\left(Y_{n}^{m}\right)_{\theta}\right|\right.\right. \\
& \left.\left.+\left|(\sin \theta)^{-1}\left(Y_{n}^{m}\right)_{\varphi}\right|\right) d \varphi\right] d \theta
\end{aligned}
$$$$
\leq C_{1}|x|\|F\|_{C^{1}(\bar{\Omega})} .
$$

To prove case (2) it remains to substitute $\left(\Delta_{S}\right)^{k} f \in C^{1}(\bar{\Omega})$ for $F$ in this estimate.

\section{Necessary Conditions for Bounded Solution}

Here we will prove of the necessity of the orthogonality conditions (10) for the existence of a bounded solution.

Proof of Theorem 3. Let $u$ be a bounded generalized solution of Problem P2. Let us fix a function $\chi(s) \in C^{\infty}(\mathbb{R})$ such that $\chi(s)=0$ for $s \leq 1$, and $\chi(s)=1$ for $s \geq 2$.

For fixed indexes $n \in \mathbb{N} \cup\{0\}, 0 \leq k \leq[n / 2], 1 \leq m \leq$ $2 n+1$, consider the functions

$$
W_{q}(x, t)=\chi(2 q(|x|-t)) \chi(q|x|) W_{k, m}^{n}(x, t),
$$

for $q \in \mathbb{N}$. Obviously, $W_{q} \in C^{\infty}(\bar{\Omega})$ vanishes on a neighborhood of $\Sigma_{2}$ and $\left(W_{q}\right)_{t}=0$ on $\Sigma_{0}$. Therefore according to Definition 2 we have

$$
\begin{gathered}
\int_{\Omega}\left\{u_{t}\left(W_{q}\right)_{t}-u_{x_{1}}\left(W_{q}\right)_{x_{1}}-u_{x_{2}}\left(W_{q}\right)_{x_{2}}-u_{x_{3}}\left(W_{q}\right)_{x_{3}}\right\} d x d t \\
=\int_{\Omega} f W_{q} d x d t
\end{gathered}
$$

and thus

$$
\begin{aligned}
& \int_{\Omega} f W_{q} d x d t \\
& \quad=\int_{\Omega} u\left\{\left(W_{q}\right)_{x_{1} x_{1}}+\left(W_{q}\right)_{x_{2} x_{2}}+\left(W_{q}\right)_{x_{3} x_{3}}-\left(W_{q}\right)_{t t}\right\} d x d t
\end{aligned}
$$

We want to prove that $\int_{\Omega} f W_{k, m}^{n} d x d t=0$ for all $n \in \mathbb{N} \cup\{0\}$, $k=0, \ldots,[n / 2], m=1, \ldots, 2 n+1$. Notice that $\left|f W_{k, m}^{n}\right| \leq$ $C|x|^{-1}$ and then the integral $\int_{\Omega}\left|f W_{k, m}^{n}\right| d x d t$ is convergent. The function $f \in C(\bar{\Omega})$ and thus

$$
\lim _{q \rightarrow+\infty} \int_{\Omega} f W_{q} d x d t=\int_{\Omega} f W_{k, m}^{n} d x d t
$$

Therefore it is sufficient to prove that when $q \rightarrow+\infty$, the right-hand side of the equality (67) tends to zero for $n \in \mathbb{N} \cup$ $\{0\}, k=0, \ldots,[n / 2], m=1, \ldots, 2 n+1$.

Using the fact that the functions $W_{k, m}^{n}$ are solutions of the homogenous wave equation in $\Omega$, straightforward computations show that

$$
\begin{aligned}
\sum_{i=1}^{3}\left(W_{q}\right)_{x_{i} x_{i}}-\left(W_{q}\right)_{t t} & \\
= & {\left[\left(W_{k, m}^{n}\right)_{r}+\left(W_{k, m}^{n}\right)_{t}+\frac{1}{r} W_{k, m}^{n}\right] 4 q \chi_{1}^{\prime} \chi_{2} } \\
& +4 q^{2} W_{k, m}^{n} \chi_{1}^{\prime} \chi_{2}^{\prime}+\left[\left(W_{k, m}^{n}\right)_{r}+\frac{1}{r} W_{k, m}^{n}\right] 2 q \chi_{1} \chi_{2}^{\prime} \\
& +q^{2} W_{k, m}^{n} \chi_{1} \chi_{2}^{\prime \prime},
\end{aligned}
$$

where $r=|x|$, while $\chi_{1}$ and $\chi_{2}$ stand for $\chi(2 q(r-t))$ and $\chi(q r)$, respectively. Expressed with spherical polar coordinates $(r, \varphi, \theta)$ in $\mathbb{R}^{3}$,

$$
\begin{gathered}
\int_{\Omega} u\left[\left(W_{q}\right)_{x_{1} x_{1}}+\left(W_{q}\right)_{x_{2} x_{2}}+\left(W_{q}\right)_{x_{3} x_{3}}-\left(W_{q}\right)_{t t}\right] d x d t \\
=\int_{\Omega}\left\{\left[\left(E_{k}^{n}\right)_{r}+\left(E_{k}^{n}\right)_{t}+\frac{1}{r} E_{k}^{n}\right] 4 q \chi_{1}^{\prime} \chi_{2}\right. \\
+q^{2} E_{k}^{n}\left(4 \chi_{1}^{\prime} \chi_{2}^{\prime}+\chi_{1} \chi_{2}^{\prime \prime}\right) \\
\left.+\left[\left(E_{k}^{n}\right)_{r}+\frac{1}{r} E_{k}^{n}\right] 2 q \chi_{1} \chi_{2}^{\prime}\right\} \\
\times u Y_{n}^{m}(\theta, \varphi) r^{2} \sin \theta d r d \varphi d \theta d t .
\end{gathered}
$$


For simplicity, we consider only one of the terms from the definition of the function $E_{k}^{n}(r, t)$ (see (6)). Let us denote

$$
w(r, t)=\frac{\left(r^{2}-t^{2}\right)^{n-k-i}}{r^{n-2 i+1}},
$$

defined in $D=\{0<t<1 / 2, t<r<1-t\}$, and consider the subsets $D_{1, q}=D \cap\{1 /(2 q)<r-t<1 / q\} \cap\{r>1 / q\}$ and $D_{2, q}=D \cap\{1 / q<r<2 / q\}$.

Since the functions $u, \chi^{\prime}, \chi^{\prime \prime}$, and $Y_{n}^{m}$ are bounded, the function $\chi^{\prime}(q r)=0$ in $D \backslash D_{2, q}$, and $\chi^{\prime}(2 q(r-t)) \chi(q r)$ is zero in $D \backslash D_{1, q}$, it is sufficient to prove that the integrals

$$
\begin{aligned}
& I_{1, q}:=q \int_{D_{1, q}}\left|w_{r}+w_{t}+r^{-1} w\right| r^{2} d r d t ; \\
& I_{2, q}:=q \int_{D_{2, q}}\left|w_{r}+r^{-1} w\right| r^{2} d r d t, \\
& I_{3, q}:=q^{2} \int_{D_{2, q}} w r^{2} d r d t
\end{aligned}
$$

tend to zero as $q \rightarrow \infty$, for $n \in \mathbb{N} \cup\{0\}, k=0, \ldots,[n / 2]$, and $i=0, \ldots, k$.

We get $\lim _{q \rightarrow \infty} I_{3, q}=0$ from the estimate $|w| \leq r^{n-2 k-1}$ in $D$ and thus

$$
\begin{aligned}
I_{3, q} & \leq q^{2} \int_{r=0}^{r=2 / q} \int_{t=0}^{t=r} r^{n-2 k+1} d t d r \\
& \leq q^{2} C \int_{0}^{2 / q} r^{2} d r \leq C_{1} q^{-1} .
\end{aligned}
$$

For $I_{2, q}$ let us compute first

$$
\begin{aligned}
\mid w_{r}+ & r^{-1} w \mid r^{2} \\
& =\frac{\left(r^{2}-t^{2}\right)^{n-k-i-1}}{r^{n-2 i}}\left[(n-2 k) r^{2}+(n-2 i) t^{2}\right] \leq C,
\end{aligned}
$$

because $n-k-i-1$ could be negative only when $n-2 k=$ $n-2 i=0$. Therefore, we have

$$
I_{2, q} \leq q \int_{r=0}^{r=2 / q} \int_{t=0}^{t=r} C d t d r \leq C_{1} q^{-1}
$$

and thus $\lim _{q \rightarrow \infty} I_{2, q}=0$

Finally, to evaluate $I_{1, q}$, we use the estimate

$$
\begin{aligned}
\mid w_{r} & +w_{t}+r^{-1} w \mid r^{2} \\
& =\frac{\left(r^{2}-t^{2}\right)^{n-k-i-1}}{r^{n-2 i}}(r-t)|(n-2 k) r-(n-2 i) t| \\
& \leq C \frac{r-t}{r},
\end{aligned}
$$

and find

$$
\begin{aligned}
I_{1, q} & \leq q C \int_{r=1 / q}^{r=1} \int_{t=r-1 / q}^{t=r} \frac{r-t}{r} d t d r \\
& \leq q^{-1} C_{1} \int_{r=1 / q}^{r=1} r^{-1} d r \leq C_{2} q^{-1} \ln q
\end{aligned}
$$

that shows that $\lim _{q \rightarrow \infty} I_{1, q}=0$.

\section{Previous Results}

In this section we quote some results from [10] that will be essentially the starting point for the proofs of Theorems 4 and 6. We start with the following uniqueness result.

Theorem 18 (see [10]). The Problem P2 has at most one generalized solution.

In [10] the right-hand side function $f$ of the wave equation (1) is fixed as a harmonic polynomial (11). Then the following existence result for the generalized solution is valid.

Theorem 19 (see [10]). Suppose that the right-hand side $f \in$ $C^{1}(\bar{\Omega})$ has the form (11) where $l \in \mathbb{N} \cup\{0\}$. Then, the unique generalized solution $u(x, t)$ of the Problem $P 2$ in $\Omega$ exists and has the form

$$
u(x, t)=\sum_{n=0}^{l} \sum_{m=1}^{2 n+1} u_{n}^{m}(|x|, t) Y_{n}^{m}(x) \in C^{2}(\bar{\Omega} \backslash O)
$$

In fact, the function $u_{n}^{m}(|x|, t)$ from (78) is the solution of a two-dimensional boundary value problem that involves only the corresponding coefficient $f_{n}^{m}(|x|, t)$ from (11). In order to formulate this BVP, it is natural to introduce polar coordinates $(r, \theta, \varphi)$ in $\mathbb{R}^{3}: r ; \theta$ and $\varphi$ are such that $0 \leq \theta<\pi$, $0 \leq \varphi<2 \pi, r>0$ and

$$
x_{1}=r \sin \theta \cos \varphi, \quad x_{2}=r \sin \theta \sin \varphi, \quad x_{3}=r \cos \theta \text {. }
$$

In the special case when $f$ has the form

$$
f(r, \theta, \varphi, t)=f_{n}^{m}(r, t) Y_{n}^{m}(\theta, \varphi),
$$

according to Theorem 19, we may look for a solution of the same form

$$
u(r, \theta, \varphi, t)=u_{n}^{m}(r, t) Y_{n}^{m}(\theta, \varphi)
$$

Then we can reduce the $(3+1)-\mathrm{D}$ Protter problem to some BVPs in $\mathbb{R}^{2}$. From the properties of the spherical functions it follows that the function $u_{n}^{m}(r, t)$ is a solution of the equation

$$
u_{r r}+\frac{2}{r} u_{r}-u_{t t}-\frac{n(n+1)}{r^{2}} u=f(r, t)
$$


with right-hand side $f(r, t):=f_{n}^{m}(r, t)$, in the domain $D=$ $\{(r, t): 0<t<1 / 2, t<r<1-t\}$, bounded by

$$
\begin{aligned}
& S_{0}=\{(r, t): t=0,0<r<1\}, \\
& S_{1}=\left\{(r, t): 0<t<\frac{1}{2}, r=1-t\right\}, \\
& S_{2}=\left\{(r, t): 0<t<\frac{1}{2}, r=t\right\} .
\end{aligned}
$$

Thus, we arrive at the next two-dimensional problems.

Problem P21. Find a solution of (82) in the domain $D$ which satisfies the boundary conditions

$$
\text { P21: }\left.\quad u_{t}\right|_{S_{0}}=0,\left.\quad u\right|_{S_{1}}=0
$$

Finally, we substitute

$$
\begin{array}{cl}
v=r u(r, t), & g=r f(r, t), \\
\xi=\frac{r+t}{2}, & \eta=\frac{r-t}{2}
\end{array}
$$

and get the following problem.

Problem P22. Find a solution of the equation

$$
v_{\xi \eta}-\frac{n(n+1)}{(\xi+\eta)^{2}} v=g
$$

in the domain $D_{1}=\left\{(\xi, \eta) \in \mathbb{R}^{2}: 0<\eta<\xi<1 / 2\right\}$ with boundary conditions

$$
\begin{array}{r}
P 22: \quad\left(\frac{\partial v}{\partial \xi}-\frac{\partial v}{\partial \eta}\right)(\eta, \eta)=0, \quad v\left(\frac{1}{2}, \eta\right)=0 \\
\text { for } \eta \in\left(0, \frac{1}{2}\right] .
\end{array}
$$

In [10] the solution of Problem P22 is constructed with the help of the Riemann's function

$$
R\left(\xi_{1}, \eta_{1} ; \xi, \eta\right)=P_{n}\left(\frac{(\xi-\eta)\left(\xi_{1}-\eta_{1}\right)+2 \xi_{1} \eta_{1}+2 \xi \eta}{\left(\xi_{1}+\eta_{1}\right)(\xi+\eta)}\right),
$$

for equation (86) found by Copson [35]. The problem is reduced to an integral equation of Volterra type. Then this integral equation is solved using some formulas from the book by Samko et al. [36] and the properties of the Mellin transform. According to formulas (23), (24), and (27) in the proof of Theorem 3.1 from [10] we can write down the following result.
Lemma 20. The solution $v(\xi, \eta)$ of Problem P22 is given by

$$
\begin{aligned}
v(\xi, \eta)= & \tau(\xi)+\int_{\xi}^{1 / 2} \tau\left(\xi_{1}\right) \frac{\partial}{\partial \xi_{1}} P_{n}\left(\frac{(\xi-\eta) \xi_{1}+2 \xi \eta}{\xi_{1}(\xi+\eta)}\right) d \xi_{1} \\
- & \int_{\xi}^{1 / 2}\left(\int_{0}^{\eta} P_{n}\left(\frac{(\xi-\eta)\left(\xi_{1}-\eta_{1}\right)+2 \xi_{1} \eta_{1}+2 \xi \eta}{\left(\xi_{1}+\eta_{1}\right)(\xi+\eta)}\right)\right. \\
& \left.\times g\left(\xi_{1}, \eta_{1}\right) d \eta_{1}\right) d \xi_{1},
\end{aligned}
$$

where

$$
\begin{aligned}
& \tau(\xi)= \int_{1 / 2}^{\xi} P_{n}\left(\frac{\xi_{1}}{\xi}\right) G\left(\xi_{1}\right) d \xi_{1} \\
& G(\xi):=\int_{\xi}^{1 / 2}\left(\int_{0}^{\xi} P_{n}\left(\frac{\xi_{1} \eta_{1}+\xi^{2}}{\xi\left(\xi_{1}+\eta_{1}\right)}\right)\right. \\
&\left.\quad \times\left(\frac{\partial}{\partial \xi_{1}}-\frac{\partial}{\partial \eta_{1}}\right) g\left(\xi_{1}, \eta_{1}\right) d \eta_{1}\right) d \xi_{1} \\
&-\int_{0}^{\xi} P_{n}\left(\frac{\eta_{1}+2 \xi^{2}}{\xi\left(2 \eta_{1}+1\right)}\right) g\left(\frac{1}{2}, \eta_{1}\right) d \eta_{1} \\
&-\int_{\xi}^{1 / 2} P_{n}\left(\frac{\xi}{\xi_{1}}\right) g\left(\xi_{1}, 0\right) d \xi_{1} .
\end{aligned}
$$

Finally, we will need the relation between the functions $E_{i}^{n}(r, t):=E_{i}^{n}(x, t)$, with $r=|x|$, defined in (6) and the Legendre polynomials $P_{n}$.

Lemma 21 (see [10]). Define the functions

$$
h_{k}(\xi, \eta)=\int_{\eta}^{\xi} s^{k} P_{n}\left(\frac{\xi \eta+s^{2}}{s(\xi+\eta)}\right) d s
$$

Then the equality

$$
r^{-1} \frac{\partial}{\partial t}\left[h_{n-2 i}\left(\frac{r+t}{2}, \frac{r-t}{2}\right)\right]=c_{i}^{n} E_{i}^{n}(r, t)
$$

holds for $i=0, \ldots,[n / 2]$ with some nonzero constants $c_{i}^{n}$.

\section{Proofs of the Main Results}

In this section we will prove Theorems 4 and 6 . The proofs are based on an asymptotic expansion formula for the solution of the two-dimensional Problem P21. In order to formulate it, let us concentrate first on Problem $P 2$ with right-hand side functions $f$ of the form

$$
f(x, t)=f_{n}^{m}(r, t) Y_{n}^{m}(\theta, \varphi),
$$

with fixed $n, m \in \mathbb{N} \cup\{0\}$ and $m \leq 2 n+1$. We will use the results stated in Section 5. The unique generalized solution $u$ of Problem $P 2$ also has the form

$$
u(x, t)=u_{n}^{m}(r, t) Y_{n}^{m}(\theta, \varphi) .
$$


The function $u_{n}^{m}(r, t)$ is the solution of Problem P21 with the function $f(r, t):=f_{n}^{m}(r, t)$ as a right-hand side in (82). We are interested in the exact behavior of $u_{n}^{m}(r, t)$ at $(0,0)$. One expects it to depend on the constants

$$
\begin{array}{r}
\beta_{k}^{n}:=\int_{0}^{1 / 2}\left(\int_{t}^{1-t} E_{k}^{n}(r, t) f(r, t) r^{2} d r\right) d t \\
\text { for } k=0, \ldots,\left[\frac{n}{2}\right]
\end{array}
$$

that correspond to $\beta_{k, m}^{n}$ defined in (13) for the Problem P2. For simplicity, denote further

$$
\begin{aligned}
& A_{0}:=\|f\|_{C(D)} ; \\
& A_{1}:=\|f\|_{C(D)}+\left\|r f_{t}\right\|_{C(D)} ; \\
& A_{2}:=\|r f\|_{C^{1}(D)}+\left\|r f_{t}\right\|_{C^{1}(D)} ; \\
& A_{3}:=\|r f\|_{C^{1}(D)}+\left\|r f_{t}\right\|_{C^{1}(D)}+\left\|r f_{t t}\right\|_{C^{1}(D)}+\left\|r^{2} f_{t r r}\right\|_{C(D)} .
\end{aligned}
$$

Theorem 22. Let $f(r, t)$ and $r f_{t}(r, t) \in C(\bar{D})$. Then the generalized solution $u(r, t)$ of Problem P21 belongs to $C^{2}(\bar{D} \backslash$ $(0,0))$ and has the following asymptotic expansion at $(0,0)$ :

$$
u(r, t)=\sum_{k=0}^{[n / 2]} r^{-1}(r+t)^{-(n-2 k)} \beta_{k}^{n} F_{k}^{n}(r, t)+F^{n}(r, t),
$$

where

(1) the functions $F_{k}^{n} \in C^{2}(\bar{D} \backslash(0,0))$ are independent of $f$, bounded, and satisfy $F_{k}^{n}(t, t) \equiv$ const $\neq 0$;

(2) the function $F^{n} \in C^{2}(\bar{D} \backslash(0,0))$ satisfies the following estimates:

$$
\begin{gathered}
\left|F^{0}(r, t)\right| \leq C\|r f\|_{C(D)}, \\
\left|\left(F^{0}\right)_{r}(r, t)\right|+\left|\left(F^{0}\right)_{t}(r, t)\right| \leq C r^{-1}\|r f\|_{C(D)},
\end{gathered}
$$

and for $n \geq 1$

$$
\left|F^{n}(r, t)\right| \leq C A_{1} n^{2}(1+|\ln r|) .
$$

If also $r f$ and $r f_{t} \in C^{1}(D)$, then

$$
\left|F^{n}(r, t)\right| \leq C r^{-1}\left(A_{0} n^{2}+A_{2}\right),
$$

and if additionally $r f_{t t} \in C^{1}(D)$ and $r^{2} f_{t r r} \in C(D)$, then

$$
\left|\left(F^{n}\right)_{r}(r, t)\right|+\left|\left(F^{n}\right)_{t}(r, t)\right| \leq C r^{-3}\left(A_{1} n^{2}+A_{3}\right),
$$

where in all inequalities the constant $C$ is independent of $n$ and $f$.

The proof of this result is quite long and technical and we leave it for Section 7. Here we will use Theorem 22 to prove Theorems 4 and 6.
Proof of Theorem 4. Assume that the right-hand side function $f$ is a harmonic polynomial (11). Then the unique generalized solution $u(x, t)$ also is a harmonic polynomial (78), according to Theorem 19. Furthermore, the functions $u_{n}^{m}(r, t)$ are solutions of Problem $P 21$ with right-hand side $f_{n}^{m}$ that can be represented as

$$
f_{n}^{m}(r, t):=\int_{0}^{\pi}\left(\int_{0}^{2 \pi} f(r, \theta, \varphi, t) Y_{n}^{m}(\theta, \varphi) d \varphi\right) \sin \theta d \theta .
$$

When $f \in C^{1}(\bar{\Omega})$ we have $f_{n}^{m} \in C^{1}(\bar{D})$ and obviously

$$
\left\|f_{n}^{m}(r, t)\right\|_{C^{1}(D)} \leq C\|f(x, t)\|_{C^{1}(\Omega)} .
$$

The definition of functions $W_{k, m}^{n}$ from Lemma 1 and (103) gives the identity

$$
\begin{gathered}
\int_{0}^{1 / 2}\left(\int_{t}^{1-t} E_{k}^{n}(r, t) f_{n}^{m}(r, t) r^{2} d r\right) d t \\
=\int_{\Omega} W_{k, m}^{n}(x, t) f(x, t) d x d t,
\end{gathered}
$$

which shows that $\beta_{k}^{n}=\beta_{k, m}^{n}$, according to their definitions (96) and (13).

Now we can apply Theorem 22 for the functions $u_{n}^{m}(r, t)$ and $f_{n}^{m}(r, t)$. Using (104) and (105) we get the expansion

$$
\begin{aligned}
u_{n}^{m}(r, t)= & \sum_{k=0}^{[n / 2]} r^{-1}(r+t)^{-(n-2 k)} \beta_{k, m}^{n} F_{k}^{n, m}(r, t) \\
& +F^{n, m}(r, t),
\end{aligned}
$$

where $\left|F^{n, m}(r, t)\right| \leq C\|f\|_{C^{1}(\Omega)},\left|F_{k}^{n, m}(r, t)\right| \leq C$, and $F_{k}^{n, m}(t, t)=$ const $\neq 0$. Summing up over $n$ and $m$ one gets the desired expansion.

Finally, to prove property (iii), let us fix a direction $(\alpha, 1):=\left(\alpha_{1}, \alpha_{2}, \alpha_{3}, 1\right)$ with $\alpha_{1}=\sin \theta_{0} \cos \varphi_{0}, \alpha_{2}=$ $\sin \theta_{0} \sin \varphi_{0}$, and $\alpha_{3}=\cos \theta_{0}$. Then for the functions $F_{k, m}^{n}$ from (16) we have

$$
F_{k, m}^{n}(\alpha t, t):=2^{(2 k-n+1) / 2} F_{k}^{n, m}(t, t) Y_{n}^{m}\left(\varphi_{0}, \theta_{0}\right) .
$$

And, thus, there are some nonzero constants $C_{p, k, m}$ such that

$$
\begin{aligned}
\lim _{t \rightarrow+0} F_{p}(\alpha t, t) & \\
& =\sum_{k=0}^{[(l-p+1) / 2]} \sum_{m=1}^{2 p+4 k-1} C_{p, k, m} \beta_{k, m}^{p+2 k-1} Y_{p+2 k-1}^{m}\left(\varphi_{0}, \theta_{0}\right) .
\end{aligned}
$$

Therefore property (iii) follows from the fact that the spherical functions $Y_{n}^{m}$ are linearly independent.

Besides Theorem 22, the proof of Theorem 6 relies on and the estimates from Section 3 for the special functions. 
Proof of Theorem 6. The function $f \in C^{6}(\bar{\Omega})$ can be represented as

$$
f(x, t)=\sum_{n=0}^{\infty} \sum_{m=1}^{2 n+1} f_{n}^{m}(|x|, t) Y_{n}^{m}(x) .
$$

The generalized solution of Problem P2 could be formally written in the form

$$
u(x, t)=\sum_{n=0}^{\infty} \sum_{m=1}^{2 n+1} u_{n}^{m}(|x|, t) Y_{n}^{m}(x),
$$

where $u_{n}^{m}(r, t)$ is the solution of Problem P21 with right-hand side $f_{n}^{m}$. We will prove that the series (110) and its derivatives are uniformly convergent in $\bar{\Omega} \cap\{r \geq \varepsilon\}$ for $\varepsilon>0$ and that $u$ is bounded.

According to Lemma 17 the series for $f$ and its first derivatives uniformly converge. For the derivatives with respect to $x_{i}$ and with respect to $r$, there holds the relation

$$
\sum_{i=1}^{3} x_{i} \frac{\partial}{\partial x_{i}} f(x, t)=\sum_{n=0}^{\infty} \sum_{m=1}^{2 n+1} r \frac{\partial}{\partial r}\left(f_{n}^{m}(r, t)\right) Y_{n}^{m}(x),
$$

and therefore $r\left(f_{n}^{m}\right)_{r} \in C(\bar{D})$. A similar argument shows that $r f_{n}^{m}, r\left(f_{n}^{m}\right)_{t}, r\left(f_{n}^{m}\right)_{t t} \in C^{1}(\bar{D})$ and $r^{2}\left(f_{n}^{m}\right)_{t r r} \in C(\bar{D})$ and we can apply Theorem 22 .

First, using Lemma 17 case (1) with $k=3$ and $k=2$, we see that for $n>0$

$$
\begin{gathered}
\left\|f_{n}^{m}\right\|_{C(D)} \leq C n^{-6}\|f\|_{C^{6}(\bar{\Omega})}, \\
\left\|r f_{n}^{m}\right\|_{C^{1}(D)}+\left\|r\left(f_{n}^{m}\right)_{t}\right\|_{C^{1}(D)} \leq C n^{-4}\|f\|_{C^{6}(\bar{\Omega})} .
\end{gathered}
$$

On the other hand, Lemma 17 case (2) with $k=2$ and $k=1$ gives

$$
\begin{gathered}
\sum_{m=1}^{2 n+1}\left\|f_{n}^{m}\right\|_{C(D)} \leq C n^{-4} r\|f\|_{C^{5}(\bar{\Omega})}, \\
\sum_{m=1}^{2 n+1}\left\|\left(f_{n}^{m}\right)_{t}\right\|_{C(D)} \leq C n^{-4} r\left\|f_{t}\right\|_{C^{5}(\bar{\Omega})}, \\
\sum_{m=1}^{2 n+1}\left(\left\|r f_{n}^{m}\right\|_{C^{1}(D)}+\left\|r\left(f_{n}^{m}\right)_{t}\right\|_{C^{1}(D)}\right. \\
\left.+\left\|r\left(f_{n}^{m}\right)_{t t}\right\|_{C^{1}(D)}+\left\|r^{2}\left(f_{n}^{m}\right)_{t r r}\right\|_{C(D)}\right) \\
\leq C n^{-2} r\|f\|_{C^{6}(\bar{\Omega})} .
\end{gathered}
$$

Next we study the series (110). Using the notations of Theorem 22, we know that when $\beta_{k}^{n}=0$ for $k=0, \ldots,[n / 2]$, the function $u_{n}^{m}(r, t)$ satisfies

$$
u_{n}^{m}(r, t)=F^{n}(r, t)
$$

and then for $n>0$ from (100), (101), and (102) we have the following estimates:

$$
\left|u_{n}^{m}(r, t)\right| \leq C n^{2}\left(\left\|f_{n}^{m}\right\|_{C(D)}+\left\|r\left(f_{n}^{m}\right)_{t}\right\|_{C(D)}\right)(1+|\ln r|),
$$

or alternatively

$$
\begin{aligned}
& \left|u_{n}^{m}(r, t)\right| \\
& \quad \leq C r^{-1}\left(n^{2}\left\|f_{n}^{m}\right\|_{C(D)}+\left\|r f_{n}^{m}\right\|_{C^{1}(D)}+\left\|r\left(f_{n}^{m}\right)_{t}\right\|_{C^{1}(D)}\right),
\end{aligned}
$$

and for the derivatives

$$
\begin{aligned}
\left|\left(u_{n}^{m}\right)_{r}(r, t)\right|+ & \left|\left(u_{n}^{m}\right)_{t}(r, t)\right| \\
\leq C r^{-3}( & n^{2}\left\|f_{n}^{m}\right\|_{C(D)}+n^{2}\left\|r\left(f_{n}^{m}\right)_{t}\right\|_{C(D)} \\
+ & \left\|r f_{n}^{m}\right\|_{C^{1}(D)}+\left\|r\left(f_{n}^{m}\right)_{t}\right\|_{C^{1}(D)} \\
& \left.+\left\|r\left(f_{n}^{m}\right)_{t t}\right\|_{C^{1}(D)}+\left\|r^{2}\left(f_{n}^{m}\right)_{t r r}\right\|_{C(D)}\right) .
\end{aligned}
$$

Then applying the estimate (49) for the spherical functions from Corollary 16 and substituting (113) and (114) in (117) we find

$$
\begin{aligned}
& \sum_{m=1}^{2 n+1}\left|u_{n}^{m} Y_{n}^{m}\right| \\
& \quad \leq C n^{-5 / 2}(1+|\ln r|) \sum_{m=1}^{2 n+1}\left(\left\|f_{n}^{m}\right\|_{C(D)}+\left\|r\left(f_{n}^{m}\right)_{t}\right\|_{C(D)}\right) \\
& \quad \leq C n^{-3 / 2}\left(\|f\|_{C^{5}(\bar{\Omega})}+\left\|f_{t}\right\|_{C^{5}(\bar{\Omega})}\right) .
\end{aligned}
$$

On the other hand, first using (118) with (112) and then (52) for the sum of derivatives of $Y_{n}^{m}$, we get

$$
\begin{aligned}
& \sum_{m=1}^{2 n+1} \sum_{|\alpha|=1}\left|u_{n}^{m} D_{(x, t)}^{\alpha} Y_{n}^{m}\right| \\
& \quad \leq C n^{-4} r^{-1}\|f\|_{C^{6}(\bar{\Omega})} \sum_{m=1}^{2 n+1} \sum_{|\alpha|=1}\left|D_{(x, t)}^{\alpha} Y_{n}^{m}\right| \\
& \leq C n^{-2} r^{-2}\|f\|_{C^{6}(\bar{\Omega})} .
\end{aligned}
$$

Combining (119) for the derivatives of $u$ with (113), (114), and (115) gives

$$
\begin{gathered}
\sum_{m=1}^{2 n+1} \sum_{|\alpha|=1}\left|Y_{n}^{m} D_{(x, t)}^{\alpha} u_{n}^{m}\right| \\
\leq C n^{5 / 2} r^{-3} \sum_{m=1}^{2 n+1}\left(\left\|f_{n}^{m}\right\|_{C(D)}+\left\|r\left(f_{n}^{m}\right)_{t}\right\|_{C(D)}\right) \\
+C n^{1 / 2} r^{-3} \sum_{m=1}^{2 n+1}\left(\left\|r f_{n}^{m}\right\|_{C^{1}(D)}+\left\|r\left(f_{n}^{m}\right)_{t}\right\|_{C^{1}(D)}\right. \\
+\left\|r\left(f_{n}^{m}\right)_{t t}\right\|_{C^{1}(D)} \\
\left.+\left\|r^{2}\left(f_{n}^{m}\right)_{t r r}\right\|_{C(D)}\right) \\
\leq C n^{-3 / 2} r^{-2}\|f\|_{C^{6}(\bar{\Omega})} .
\end{gathered}
$$


For the case $n=0$ we have $Y_{0}^{1}=$ const and representation (103) shows that $\left|f_{0}^{1}\right| \leq C\|f\|_{C(\bar{\Omega})}$. Therefore Theorem 22 gives

$$
\begin{gathered}
\left|u_{0}^{1} Y_{0}^{1}\right| \leq C\|f\|_{C(\bar{\Omega})}, \\
\sum_{|\alpha|=1}\left|D_{(x, t)}^{\alpha} u_{0}^{1} Y_{0}^{1}\right| \leq C r^{-1}\|f\|_{C(\bar{\Omega})} .
\end{gathered}
$$

After this preparation, we are ready to estimate the Fourier series (110) and its first derivatives:

$$
\begin{aligned}
& |u(x, t)| \\
& \leq C\|f\|_{C(\bar{\Omega})}+C\left(\|f\|_{C^{5}(\bar{\Omega})}+\left\|f_{t}\right\|_{C^{5}(\bar{\Omega})}\right) \sum_{n=1}^{\infty} n^{-2} \\
& \leq C\left(\|f\|_{C^{5}(\bar{\Omega})}+\left\|f_{t}\right\|_{C^{5}(\bar{\Omega})}\right), \\
& \sum_{|\alpha|=1}\left|D_{(x, t)}^{\alpha} u(x, t)\right| \\
& \leq C r^{-1}\|f\|_{C(\bar{\Omega})}+C r^{-2}\|f\|_{C^{6}(\bar{\Omega})} \sum_{n=1}^{\infty} n^{-3 / 2} \\
& \quad+C r^{-2}\|f\|_{C^{6}(\bar{\Omega})} \sum_{n=1}^{\infty} n^{-2} \\
& \leq C r^{-2}\|f\|_{C^{6}(\bar{\Omega})} .
\end{aligned}
$$

Therefore we have $u \in C^{1}(\bar{\Omega} \backslash O)$ since for each fixed $\varepsilon>0$ the series (110) uniformly converges in the set $\bar{\Omega} \cap\{r \geq \varepsilon\}$ and the same holds for its first derivatives.

Finally, we will prove that the function $u(x, t)$ defined as the series (110) is the generalized solution of Problem $P 2$. First, notice that the function $u_{n}^{m}(|x|, t) Y_{n}^{m}(x)$ is the generalized solution of Protter Problem $P 2$ with right-hand side function $f_{n}^{m}(|x|, t) Y_{n}^{m}(x)$. Thus $u \in C^{1}(\bar{\Omega} \backslash O)$ satisfies the boundary conditions $\left.u_{t}\right|_{\Sigma_{0}}=0$ and $\left.u\right|_{\Sigma_{1}}=0$ just like all the terms $u_{n}^{m} Y_{n}^{m}$. The proof of case (2) from Definition 2 is straightforward; for a test function $w$ and $l \in \mathbb{N}$ we have

$$
\begin{aligned}
\int_{\Omega} \sum_{n=0}^{l} \sum_{m=1}^{2 n+1}\{ & \left\{u_{n}^{m} Y_{n}^{m}\right)_{t} w_{t}-\left(u_{n}^{m} Y_{n}^{m}\right)_{x_{1}} w_{x_{1}} \\
- & \left(u_{n}^{m} Y_{n}^{m}\right)_{x_{2}} w_{x_{2}}-\left(u_{n}^{m} Y_{n}^{m}\right)_{x_{3}} w_{x_{3}} \\
- & \left.f_{n}^{m} Y_{n}^{m} w\right\} d x d t=0,
\end{aligned}
$$

and the uniform convergence in $\Omega \cap \operatorname{supp}(w)$ of the series (110) and its first derivatives allow us to take the limit $l \rightarrow \infty$ in this equality. Therefore

$$
\int_{\Omega}\left\{u_{t} w_{t}-u_{x_{1}} w_{x_{1}}-u_{x_{2}} w_{x_{2}}-u_{x_{3}} w_{x_{3}}-f w\right\} d x d t=0,
$$

and we see that $u(x, t)$ is the generalized solution of Problem $P 2$ with right-hand side $f(x, t)$.

\section{Proof of the Asymptotic Expansion in the Two-Dimensional Case}

The proof of Theorem 22 is based on the results stated in Section 5. In particular, according to Lemma 20, the solution $u(r, t)$ of Problem P21 can be constructed with help of the substitutions $\xi=(r+t) / 2, \eta=(r-t) / 2$ as

$$
u(\xi+\eta, \xi-\eta)=(\xi+\eta)^{-1} v(\xi, \eta),
$$

where $v(\xi, \eta)$ is defined by the formulas (89), (90), (91), and

$$
g(\xi, \eta)=(\xi+\eta) f(\xi+\eta, \xi-\eta) .
$$

One can see that generally the integral in (90) blows up when $\xi$ approaches 0 , and thus $v(\xi, \eta)$ has singularity at $(0,0)$ even for smooth functions $g(\xi, \eta)$.

Proof of Theorem 22. The proof is as follows.

(A) Proof of the Asymptotic Formula. We will study the behavior of the function

$$
v(\xi, \eta)=(\xi+\eta) u(\xi+\eta, \xi-\eta),
$$

given by the integral representation (89) from Lemma 20 . The smoothness of $v(\xi, \eta)$ in (89) away from the point $(0,0)$ follows directly from the smoothness of the function $G(\xi)$. Next we will derive the asymptotic expansion of $v(\xi, \eta)$ at $(0,0)$.

First we will find the relation between the constants $\beta_{k}^{n}$ and the function $G(\xi)$ defined by (91). Let us compute the integral

$$
\begin{aligned}
& \int_{0}^{1 / 2} \xi^{k} G(\xi) d \xi \\
& =\int_{0}^{1 / 2} \int_{\xi}^{1 / 2} \int_{0}^{\xi} \xi^{k} P_{n}\left(\frac{\xi_{1} \eta_{1}+\xi^{2}}{\xi\left(\xi_{1}+\eta_{1}\right)}\right)\left(\frac{\partial}{\partial \xi_{1}}-\frac{\partial}{\partial \eta_{1}}\right) \\
& \quad \times g\left(\xi_{1}, \eta_{1}\right) d \eta_{1} d \xi_{1} d \xi \\
& \quad-\int_{0}^{1 / 2} \int_{0}^{\xi} \xi^{k} P_{n}\left(\frac{\eta_{1}+2 \xi^{2}}{\xi\left(2 \eta_{1}+1\right)}\right) g\left(\frac{1}{2}, \eta_{1}\right) d \eta_{1} d \xi \\
& \quad-\int_{0}^{1 / 2} \int_{\xi}^{1 / 2} \xi^{k} P_{n}\left(\frac{\xi}{\xi_{1}}\right) g\left(\xi_{1}, 0\right) d \xi_{1} d \xi \\
& =\int_{0}^{1 / 2} \int_{0}^{\xi_{1}}\left(\int_{\eta_{1}}^{\xi_{1}} \xi^{k} P_{n}\left(\frac{\xi_{1} \eta_{1}+\xi^{2}}{\xi\left(\xi_{1}+\eta_{1}\right)}\right) d \xi\right) \\
& \quad \times\left(\frac{\partial}{\partial \xi_{1}}-\frac{\partial}{\partial \eta_{1}}\right) g\left(\xi_{1}, \eta_{1}\right) d \eta_{1} d \xi_{1} \\
& \quad-\int_{0}^{1 / 2}\left(\int_{\eta_{1}}^{1 / 2} \xi^{k} P_{n}\left(\frac{\eta_{1}+2 \xi^{2}}{\xi\left(2 \eta_{1}+1\right)}\right) d \xi\right) g\left(\frac{1}{2}, \eta_{1}\right) d \eta_{1} \\
& \quad-\int_{0}^{1 / 2}\left(\int_{0}^{\xi_{1}} \xi^{k} P_{n}\left(\frac{\xi}{\xi_{1}}\right) d \xi\right) g\left(\xi_{1}, 0\right) d \xi_{1}
\end{aligned}
$$




$$
\begin{aligned}
=- & \int_{0}^{1 / 2} \int_{0}^{\xi_{1}}\left(\frac{\partial}{\partial \xi_{1}}-\frac{\partial}{\partial \eta_{1}}\right) \\
& \quad \times\left(\int_{\eta_{1}}^{\xi_{1}} \xi^{k} P_{n}\left(\frac{\xi_{1} \eta_{1}+\xi^{2}}{\xi\left(\xi_{1}+\eta_{1}\right)}\right) d \xi\right) g\left(\xi_{1}, \eta_{1}\right) d \eta_{1} d \xi_{1} \\
= & -\int_{0}^{1 / 2} \int_{0}^{\xi_{1}}\left(\frac{\partial}{\partial \xi_{1}}-\frac{\partial}{\partial \eta_{1}}\right) h_{k}\left(\xi_{1}, \eta_{1}\right) g\left(\xi_{1}, \eta_{1}\right) d \eta_{1} d \xi_{1} .
\end{aligned}
$$

Now we can apply Lemma 21 to get

$$
\left.\left(\frac{\partial}{\partial \xi}-\frac{\partial}{\partial \eta}\right) h_{n-2 i}(\xi, \eta)\right|_{\substack{\xi=(r+t) / 2 \\ \eta=(r-t) / 2}}=c_{i}^{n} r E_{i}^{n}(r, t),
$$

and we conclude that

$$
\begin{aligned}
\int_{0}^{1 / 2} & \xi^{n-2 i} G(\xi) d \xi \\
& =-\int_{0}^{1 / 2}\left(\int_{0}^{\xi}\left(\frac{\partial}{\partial \xi}-\frac{\partial}{\partial \eta}\right) h_{n-2 i}^{n}(\xi, \eta) g(\xi, \eta) d \eta\right) d \xi \\
& =-c_{i}^{n} \int_{0}^{1 / 2}\left(\int_{t}^{1-t} E_{i}^{n}(r, t) f(r, t) r^{2} d r\right) d t=-c_{i}^{n} \beta_{i}^{n} .
\end{aligned}
$$

Next we consider the case $n \geq 1$. The simpler case $n=0$ will be discussed separately later in the proof.

Let us expand the Legendre polynomial $P_{n}$ in formula (90) using (27):

$$
P_{n}(x)=\sum_{k=0}^{[n / 2]} a_{2 k} x^{n-2 k}
$$

where $a_{2 k} \neq 0$. For $\tau(\xi)$ we get

$$
\begin{aligned}
\tau(\xi) & =\int_{1 / 2}^{\xi} P_{n}\left(\frac{\xi_{1}}{\xi}\right) G\left(\xi_{1}\right) d \xi_{1} \\
& =\sum_{k=0}^{[n / 2]} a_{2 k} \xi^{-n+2 k} \int_{1 / 2}^{\xi} \xi_{1}^{n-2 k} G\left(\xi_{1}\right) d \xi_{1} .
\end{aligned}
$$

Applying (132) we find

$$
\begin{aligned}
\tau(\xi) & =\sum_{k=0}^{[n / 2]} a_{2 k} \xi^{-n+2 k}\left(c_{i}^{n} \beta_{i}^{n}+\int_{0}^{\xi} \xi_{1}^{n-2 k} G\left(\xi_{1}\right) d \xi_{1}\right) \\
& =\sum_{k=0}^{[n / 2]} a_{2 k} c_{i}^{n} \beta_{i}^{n} \xi^{-n+2 k}+\psi(\xi),
\end{aligned}
$$

where

$$
\begin{aligned}
\psi(\xi) & =\sum_{k=0}^{[n / 2]} a_{2 k} \xi^{-n+2 k} \int_{0}^{\xi} \xi_{1}^{n-2 k} G\left(\xi_{1}\right) d \xi_{1} \\
& =\int_{0}^{\xi} P_{n}\left(\frac{\xi_{1}}{\xi}\right) G\left(\xi_{1}\right) d \xi_{1} .
\end{aligned}
$$

Now, we want to estimate the function $\psi(\xi)$. First, let us look more carefully to the representation (91) of the function $G(\xi)$ with $g=r f(r, t)$ :

$$
\begin{aligned}
G(\xi)= & \int_{\xi}^{1 / 2}\left(\int_{0}^{\xi} P_{n}\left(\frac{\xi_{1} \eta_{1}+\xi^{2}}{\xi\left(\xi_{1}+\eta_{1}\right)}\right)\right. \\
& \left.\times\left(\frac{\partial}{\partial \xi_{1}}-\frac{\partial}{\partial \eta_{1}}\right) g\left(\xi_{1}, \eta_{1}\right) d \eta_{1}\right) d \xi_{1} \\
& -\int_{0}^{\xi} P_{n}\left(\frac{\eta_{1}+2 \xi^{2}}{\xi\left(2 \eta_{1}+1\right)}\right) g\left(\frac{1}{2}, \eta_{1}\right) d \eta_{1} \\
& -\int_{\xi}^{1 / 2} P_{n}\left(\frac{\xi}{\xi_{1}}\right) g\left(\xi_{1}, 0\right) d \xi_{1} .
\end{aligned}
$$

Since all the arguments of $P_{n}$ here are in the interval $[0,1]$ and $\left|P_{n}(x)\right| \leq 1$ for $x \in[0,1]$, it is obvious that

$$
|G(\xi)| \leq C A_{1},
$$

and therefore

$$
|\psi(\xi)| \leq C A_{1} \xi,
$$

where the constant $C$ is independent of $n$ and $f$.

We will need also a more accurate estimate for $\psi(\xi)$ with higher power of $\xi$. The first two integrals in (137) are bounded by $2 A_{1} \xi$. For the last term

$$
J_{n}(\xi):=\int_{\xi}^{1 / 2} P_{n}\left(\frac{\xi}{\xi_{1}}\right) g\left(\xi_{1}, 0\right) d \xi_{1}
$$

more computations are required. First, using the estimate (32) from Lemma 14, we have

$$
\begin{aligned}
& \left|J_{n}(\xi)-J_{n}(0)\right| \\
& \quad=\left|\int_{\xi}^{1 / 2} P_{n}\left(\frac{\xi}{\xi_{1}}\right) g\left(\xi_{1}, 0\right) d \xi_{1}-P_{n}(0) \int_{0}^{1 / 2} g\left(\xi_{1}, 0\right) d \xi_{1}\right| \\
& \quad \leq n \int_{\xi}^{1 / 2} \frac{\xi}{\xi_{1}}\left|g\left(\xi_{1}, 0\right)\right| d \xi_{1}+\left|P_{n}(0) \int_{0}^{\xi} g\left(\xi_{1}, 0\right) d \xi_{1}\right| \\
& \quad \leq C A_{1} n \xi^{\varepsilon},
\end{aligned}
$$

with some $\varepsilon \in(0,1)$ and the constant $C$ is independent of $n$. Therefore

$$
\left|G(\xi)+J_{n}(0)\right| \leq C A_{1} n \xi^{\varepsilon} .
$$

Now, notice that the value of $\psi(\xi)$ will not change if we add $J_{n}(0)$ to $G(\xi)$. This is based on the equality

$$
\begin{aligned}
& \int_{0}^{\xi} P_{n}\left(\frac{\xi_{1}}{\xi}\right) J_{n}(0) d \xi_{1} \\
& \quad \equiv P_{n}(0) \int_{0}^{1 / 2} g\left(\xi_{1}, 0\right) d \xi_{1} \int_{0}^{\xi} P_{n}\left(\frac{\xi_{1}}{\xi}\right) d \xi_{1}=0
\end{aligned}
$$

that holds, because when $n$ is odd number $P_{n}(0)=0$, while for even indices $n, n>0$, the polynomial $P_{n}$ is an even 
function and by the definition of the Legendre polynomials (see (27)) it follows that

$$
\begin{aligned}
\int_{0}^{\xi} P_{n}\left(\frac{\xi_{1}}{\xi}\right) d \xi_{1} & =\xi \int_{0}^{1} P_{n}(t) d t=\frac{1}{2} \xi \int_{-1}^{1} P_{n}(t) d t \\
& =c \xi \int_{-1}^{1} \frac{d^{n}}{d t^{n}}\left\{\left(1-t^{2}\right)^{n}\right\} d t=0 .
\end{aligned}
$$

Thus

$$
\begin{aligned}
\psi(\xi) & =\psi(\xi)+\int_{0}^{\xi} P_{n}\left(\frac{\xi_{1}}{\xi}\right) J_{n}(0) d \xi_{1} \\
& =\int_{0}^{\xi} P_{n}\left(\frac{\xi_{1}}{\xi}\right)\left[G\left(\xi_{1}\right)+J_{n}(0)\right] d \xi_{1}
\end{aligned}
$$

and we can apply (142) to conclude that for $n>0$ there is $\varepsilon>0$ and a constant $C$ independent of $n$ and $f$, such that

$$
|\psi(\xi)| \leq A_{1} C n \xi^{1+\varepsilon}
$$

Now we apply the expansion (135) of $\tau(\xi)$ in the definition (89) of $v(\xi, \eta)$ and find that

$$
\begin{aligned}
v(\xi, \eta)= & \sum_{k=0}^{[n / 2]} a_{2 k} c_{k}^{n} \beta_{k}^{n} \xi^{-n+2 k}+\psi(\xi) \\
& +\int_{\xi}^{1 / 2} \tau\left(\xi_{1}\right) \frac{\partial}{\partial \xi_{1}} P_{n}\left(\frac{(\xi-\eta) \xi_{1}+2 \xi \eta}{\xi_{1}(\xi+\eta)}\right) d \xi_{1} \\
& +F_{1}(\xi, \eta)
\end{aligned}
$$

where the function $F_{1}$ is smooth (see (89)) and $\left|F_{1}(\xi, \eta)\right| \leq$ $A_{1} C \xi$. Consider the term

$$
\begin{aligned}
\int_{\xi}^{1 / 2} & \tau\left(\xi_{1}\right) \frac{\partial}{\partial \xi_{1}} P_{n}\left(\frac{(\xi-\eta) \xi_{1}+2 \xi \eta}{\xi_{1}(\xi+\eta)}\right) d \xi_{1} \\
= & \sum_{k=0}^{[n / 2]} a_{2 k} c_{k}^{n} \beta_{k}^{n} \int_{\xi}^{1 / 2} \xi_{1}^{-n+2 k} \frac{\partial}{\partial \xi_{1}} P_{n}\left(\frac{(\xi-\eta) \xi_{1}+2 \xi \eta}{\xi_{1}(\xi+\eta)}\right) d \xi_{1} \\
& +\int_{\xi}^{1 / 2} \psi\left(\xi_{1}\right) \frac{\partial}{\partial \xi_{1}} P_{n}\left(\frac{(\xi-\eta) \xi_{1}+2 \xi \eta}{\xi_{1}(\xi+\eta)}\right) d \xi_{1} .
\end{aligned}
$$

According to (146) the last integral is bounded:

$$
\begin{aligned}
& \left|\int_{\xi}^{1 / 2} \xi_{1}^{1+\varepsilon} \frac{\partial}{\partial \xi_{1}} P_{n}\left(\frac{(\xi-\eta) \xi_{1}+2 \xi \eta}{\xi_{1}(\xi+\eta)}\right) d \xi_{1}\right| \\
& \quad \leq \frac{C \xi \eta}{\xi+\eta} \int_{\xi}^{1 / 2} \xi_{1}^{\varepsilon-1} d \xi_{1} \leq C^{\prime} \xi .
\end{aligned}
$$

Thus, we find the expansion

$$
v(\xi, \eta)=\sum_{k=0}^{n / 2} a_{2 k} c_{k}^{n} \beta_{k}^{n} \xi^{-n+2 k} G_{k}^{n}(\xi, \eta)+\psi_{1}(\xi, \eta),
$$

where

$$
\begin{aligned}
G_{k}^{n}(\xi, \eta):= & +\xi^{n-2 k} \\
& \times \int_{\xi}^{1 / 2} \xi_{1}^{-n+2 k} \frac{\partial}{\partial \xi_{1}} P_{n}\left(\frac{(\xi-\eta) \xi_{1}+2 \xi \eta}{\xi_{1}(\xi+\eta)}\right) d \xi_{1}
\end{aligned}
$$

and $\left|\psi_{1}(\xi, \eta)\right| \leq A_{1} C \xi$. Let us point out that $G_{k}^{n}(\xi, 0)=1$, while the fact that the functions $G_{k}^{n}$ are bounded follows from the estimate

$$
\left|\int_{\xi}^{1 / 2} \xi_{1}^{-n+2 k} \frac{\partial}{\partial \xi_{1}} P_{n}\left(\frac{(\xi-\eta) \xi_{1}+2 \xi \eta}{\xi_{1}(\xi+\eta)}\right) d \xi_{1}\right| \leq C \xi^{-n+2 k}
$$

The representation (150) holds for $n>0$, while when $n=0$ we have simply $P_{0} \equiv 1$ and can substitute it in formulas (89), (90), and (91) for the solution $v$ of Problem $P 22$. Straightforward computations lead to

$$
\begin{aligned}
v(\xi, \eta)= & a_{0} c_{0}^{0} \beta_{0}^{0} \\
& +\int_{0}^{\xi} G\left(\xi_{1}\right) d \xi_{1}-\int_{\xi}^{1 / 2}\left(\int_{0}^{\eta} g\left(\xi_{1}, \eta_{1}\right) d \eta_{1}\right) d \xi_{1},
\end{aligned}
$$

where

$$
G(\xi):=-\int_{0}^{\xi} g\left(\xi, \eta_{1}\right) d \eta_{1}-\int_{\xi}^{1 / 2} g\left(\xi_{1}, \xi\right) d \xi_{1} .
$$

Therefore, the representation (150) obviously stays true in the case $n=0$.

Finally, let us return to the generalized solution $u(r, t)$ of Problem $P 21$ and to the coordinates $r$ and $t$ :

$$
\begin{aligned}
u(r, t) & =r^{-1} v\left(\frac{r+t}{2}, \frac{r-t}{2}\right) \\
& =r^{-1} \sum_{k=0}^{[n / 2]} \beta_{k}^{n}(r+t)^{-n+2 k} F_{k}^{n}(r, t)+F^{n}(r, t) .
\end{aligned}
$$

Here, the function $F^{n}(r, t)$ is given by

$$
F^{n}(r, t):=r^{-1} \psi_{1}\left(\frac{r+t}{2}, \frac{r-t}{2}\right),
$$

and therefore $\left|F^{n}(r, t)\right| \leq C A_{1}$, while functions $F_{k}^{n}(r, t)$, defined by

$$
F_{k}^{n}(r, t):=2^{n-2 k} a_{2 k} c_{k}^{n} G_{k}^{n}\left(\frac{r+t}{2}, \frac{r-t}{2}\right),
$$

are independent of $f$ and are obviously bounded. To complete the proof of case (1), notice that this definition gives

$$
F_{k}^{n}(t, t)=2^{n-2 k} a_{2 k} c_{k}^{n} \neq 0 .
$$

(B) Proof of the Estimates of $F^{n}$. Next, for $n \geq 1$, we will estimate the function $F^{n}$ and its first derivatives. 
First, we will study the behavior of $F^{n}$ at $(0,0)$. The function $F^{n}(r, t)$ is given by (156), where $\psi_{1}(\xi, \eta)$ is defined in (150) as

$$
\begin{aligned}
& \psi_{1}(\xi, \eta)= \psi(\xi) \\
&+\int_{\xi}^{1 / 2} \psi\left(\xi_{1}\right) \frac{\partial}{\partial \xi_{1}} P_{n}\left(\frac{(\xi-\eta) \xi_{1}+2 \xi \eta}{\xi_{1}(\xi+\eta)}\right) d \xi_{1} \\
&+F_{1}(\xi, \eta), \\
& F_{1}(\xi, \eta)=-\int_{\xi}^{1 / 2}\left(\int_{0}^{\eta} P_{n}\right. \\
& \quad \times\left(\frac{(\xi-\eta)\left(\xi_{1}-\eta_{1}\right)+2 \xi_{1} \eta_{1}+2 \xi \eta}{\left(\xi_{1}+\eta_{1}\right)(\xi+\eta)}\right) \\
&\left.\quad \times g\left(\xi_{1}, \eta_{1}\right) d \eta_{1}\right) d \xi_{1} .
\end{aligned}
$$

Since the argument of the Legendre polynomial $P_{n}$ in (160) varies in the interval $[0,1]$,

$$
\left|F_{1}(\xi, \eta)\right| \leq C A_{0} \xi
$$

where the constant $C$ is independent of $n$ and $f$. Thus applying the estimate (139) for $\psi$ we get

$$
\begin{aligned}
\left|\psi_{1}(\xi, \eta)\right| \leq & C A_{1} \xi \\
& +C A_{1} n^{2} \int_{\xi}^{1 / 2} \frac{\xi \eta}{\xi_{1}(\xi+\eta)} d \xi_{1}+C A_{1} \xi \\
\leq & C_{1} A_{1} n^{2} \xi|\ln \xi| .
\end{aligned}
$$

Therefore

$$
\left|F^{n}(r, t)\right| \leq C_{2} A_{1} n^{2}(1+|\ln r|),
$$

where the constant $C_{2}$ is independent of $n$ and $f$; that is, (100) holds.

Now we consider the derivatives of $F^{n}$. Thus we need to evaluate the derivatives of $\psi_{1}(\xi, \eta)$; integrating (159) by parts we get

$$
\begin{aligned}
\psi_{1}(\xi, \eta)= & P_{n}\left(\frac{\xi-\eta+4 \xi \eta}{\xi+\eta}\right) \psi\left(\frac{1}{2}\right) \\
& -\int_{\xi}^{1 / 2} P_{n}\left(\frac{(\xi-\eta) \xi_{1}+2 \xi \eta}{\xi_{1}(\xi+\eta)}\right) \psi^{\prime}\left(\xi_{1}\right) d \xi_{1} \\
& +F_{1}(\xi, \eta) .
\end{aligned}
$$

For $\psi^{\prime}(\xi)$ defined by (136), using (138), we find

$$
\begin{aligned}
\left|\psi^{\prime}(\xi)\right| & =\left|G(\xi)-\int_{0}^{\xi} P_{n}^{\prime}\left(\frac{\xi_{1}}{\xi}\right) \frac{\xi_{1}}{\xi^{2}} G\left(\xi_{1}\right) d \xi_{1}\right| \\
& \leq C n^{2} \max |G(\xi)| \leq C A_{1} n^{2},
\end{aligned}
$$

where the constant $C$ is independent of $n$ and $f$. In fact, we can remove the coefficient $n^{2}$ here for smoother functions $g$. In order to do this, let us rewrite $\psi^{\prime}$ as

$$
\psi^{\prime}(\xi)=\xi^{-1} \int_{0}^{\xi} P_{n}\left(\frac{\xi_{1}}{\xi}\right) \frac{\partial}{\partial \xi_{1}}\left\{\xi_{1} G\left(\xi_{1}\right)\right\} d \xi_{1}
$$

For $\left(\psi_{1}\right)_{\xi}$ and $\left(\psi_{1}\right)_{\eta}$ we will need also $\psi^{\prime \prime}$ :

$$
\psi^{\prime \prime}(\xi)=\xi^{-2} \int_{0}^{\xi} P_{n}\left(\frac{\xi_{1}}{\xi}\right) \frac{\partial^{2}}{\partial \xi_{1}^{2}}\left\{\xi_{1} G\left(\xi_{1}\right)\right\} d \xi_{1} .
$$

Then estimates for the derivatives of $G(\xi)$ are required:

$$
\begin{aligned}
G^{\prime}(\xi)= & -\int_{0}^{\xi} \frac{\partial}{\partial \xi} g\left(\xi, \eta_{1}\right) d \eta_{1}-\int_{\xi}^{1 / 2} \frac{\partial}{\partial \xi} g\left(\xi_{1}, \xi\right) d \xi_{1} \\
& -\int_{0}^{\xi} P_{n}^{\prime}\left(\frac{\eta_{1}+2 \xi^{2}}{\xi\left(2 \eta_{1}+1\right)}\right) \frac{2 \xi^{2}-\eta_{1}}{\xi^{2}\left(2 \eta_{1}+1\right)} g\left(\frac{1}{2}, \eta_{1}\right) d \eta_{1} \\
& -\int_{\xi}^{1 / 2} P_{n}^{\prime}\left(\frac{\xi}{\xi_{1}}\right) \frac{g\left(\xi_{1}, 0\right)}{\xi_{1}} d \xi_{1} \\
& +\int_{\xi}^{1 / 2}\left(\int_{0}^{\xi} \frac{\partial}{\partial \xi} P_{n}\left(\frac{\xi_{1} \eta_{1}+\xi^{2}}{\xi\left(\xi_{1}+\eta_{1}\right)}\right)\right. \\
& \left.\times\left(\frac{\partial}{\partial \xi_{1}}-\frac{\partial}{\partial \eta_{1}}\right) g\left(\xi_{1}, \eta_{1}\right) d \eta_{1}\right) d \xi_{1} .
\end{aligned}
$$

Notice that for the argument of $P_{n}$ in the last term

$$
\left(\xi \frac{\partial}{\partial \xi}+\xi_{1} \frac{\partial}{\partial \xi_{1}}+\eta_{1} \frac{\partial}{\partial \eta_{1}}\right) \frac{\xi_{1} \eta_{1}+\xi^{2}}{\xi\left(\xi_{1}+\eta_{1}\right)}=0
$$

since the function is homogenous. Therefore we can replace there the derivative with respect to $\xi$ with $\left[-\xi_{1} \partial / \partial \xi_{1}-\right.$ $\left.\eta_{1} \partial / \partial \eta_{1}\right] / \xi$. Integrating by parts we find 


$$
\begin{aligned}
G^{\prime}(\xi)= & -g\left(\frac{1}{2}, \xi\right)-\left.\frac{1}{2 \xi} \int_{0}^{\xi} P_{n}\left(\frac{\eta_{1}+2 \xi^{2}}{\xi\left(1+2 \eta_{1}\right)}\right)\left(\frac{\partial}{\partial \xi_{1}}-\frac{\partial}{\partial \eta_{1}}\right) g\left(\xi_{1}, \eta_{1}\right)\right|_{\xi_{1}=1 / 2} d \eta_{1} \\
& +g(\xi, 0)-\int_{\xi}^{1 / 2} P_{n}^{\prime}\left(\frac{\xi}{\xi_{1}}\right) \frac{g\left(\xi_{1}, 0\right)}{\xi_{1}} d \xi_{1}-\int_{0}^{\xi} P_{n}^{\prime}\left(\frac{\eta_{1}+2 \xi^{2}}{\xi\left(2 \eta_{1}+1\right)}\right) \frac{\left(2 \xi^{2}-\eta_{1}\right) g\left(1 / 2, \eta_{1}\right)}{\xi^{2}\left(2 \eta_{1}+1\right)} d \eta_{1} \\
& +\frac{1}{\xi} \int_{\xi}^{1 / 2}\left(\int_{0}^{\xi} P_{n}\left(\frac{\xi_{1} \eta_{1}+\xi^{2}}{\xi\left(\xi_{1}+\eta_{1}\right)}\right)\left(2+\xi_{1} \frac{\partial}{\partial \xi_{1}}+\eta_{1} \frac{\partial}{\partial \eta_{1}}\right)\left(\frac{\partial}{\partial \xi_{1}}-\frac{\partial}{\partial \eta_{1}}\right) g\left(\xi_{1}, \eta_{1}\right) d \eta_{1}\right) d \xi_{1} .
\end{aligned}
$$

Recall that $g(\xi, \eta)=r f(r, t)$, and thus $g(\xi, 0) / \xi=f(r, r)$ and in the last integral we have

$$
\begin{gathered}
\left.\left(\xi \frac{\partial}{\partial \xi}+\eta \frac{\partial}{\partial \eta}\right)\left(\frac{\partial}{\partial \xi}-\frac{\partial}{\partial \eta}\right) g(\xi, \eta)\right|_{\substack{\xi=(r+t) / 2 \\
\eta=(r-t) / 2}} \\
=\left(r \frac{\partial}{\partial r}+t \frac{\partial}{\partial t}\right) r f_{t}(r, t) .
\end{gathered}
$$

Hence, using (31) from Lemma 14, we get that

$$
\left|G^{\prime}(\xi)\right| \leq C A_{0} n^{2}+C A_{2} .
$$

Analogously for $G^{\prime \prime}(\xi)$, differentiating one more time the expression for $G^{\prime}(\xi)$, applying again (169) in the last integral, and integrating by parts the last three terms, we find the estimate

$$
\left|G^{\prime \prime}(\xi)\right| \leq C A_{1} n^{2} \xi^{-1}(1-2 \xi)^{-1}+C A_{3} \xi^{-1} .
$$

Applying (138), (172), and (173) to $\psi$ and $\psi^{\prime}$ in (166) and (167) we find

$$
\begin{aligned}
& \left|\psi^{\prime}(\xi)\right| \leq C A_{0} n^{2}+C A_{2}, \\
& \left|\psi^{\prime \prime}(\xi)\right| \leq C A_{1} n^{2} \xi^{-2}|\ln (1-2 \xi)|+C A_{3} \xi^{-1} .
\end{aligned}
$$

To evaluate the derivatives of $\psi_{1}$ we need also to study the derivatives of $F_{1}$ defined by (160):

$$
\begin{aligned}
\left(F_{1}\right)_{\eta}= & -\int_{\xi}^{1 / 2} g\left(\xi_{1}, \eta\right) d \xi_{1} \\
& +\int_{\xi}^{1 / 2}\left(\int_{0}^{\eta} P_{n}^{\prime}\left(\frac{(\xi-\eta)\left(\xi_{1}-\eta_{1}\right)+2 \xi_{1} \eta_{1}+2 \xi \eta}{\left(\xi_{1}+\eta_{1}\right)(\xi+\eta)}\right)\right. \\
& \left.\times \frac{2\left(\xi_{1}-\xi\right)\left(\xi+\eta_{1}\right)}{\left(\xi_{1}+\eta_{1}\right)(\xi+\eta)^{2}} g\left(\xi_{1}, \eta_{1}\right) d \eta_{1}\right) d \xi_{1} ; \\
\left(F_{1}\right)_{\xi}=\int_{0}^{\eta} g\left(\xi, \eta_{1}\right) d \eta_{1} & +\int_{\xi}^{1 / 2}\left(\int_{0}^{\eta} P_{n}^{\prime}\left(\frac{(\xi-\eta)\left(\xi_{1}-\eta_{1}\right)+2 \xi_{1} \eta_{1}+2 \xi \eta}{\left(\xi_{1}+\eta_{1}\right)(\xi+\eta)}\right)\right. \\
& \left.\times \frac{2\left(\xi_{1}+\eta\right)\left(\eta-\eta_{1}\right)}{\left(\xi_{1}+\eta_{1}\right)(\xi+\eta)^{2}} g\left(\xi_{1}, \eta_{1}\right) d \eta_{1}\right) d \xi_{1} .
\end{aligned}
$$

Notice that in these integrals $0 \leq \eta_{1} \leq \eta \leq \xi \leq \xi_{1}$ and therefore

$$
\begin{aligned}
& \frac{\left(\xi_{1}-\xi\right)\left(\xi+\eta_{1}\right)}{\left(\xi_{1}+\eta_{1}\right)(\xi+\eta)^{2}} \leq \frac{1}{\xi+\eta}, \\
& \frac{\left(\xi_{1}+\eta\right)\left(\eta-\eta_{1}\right)}{\left(\xi_{1}+\eta_{1}\right)(\xi+\eta)^{2}} \leq \frac{1}{\xi+\eta} .
\end{aligned}
$$

Then it follows from Lemma 14 that

$$
\left|\left(F_{1}\right)_{\xi}(\xi, \eta)\right|+\left|\left(F_{1}\right)_{\eta}(\xi, \eta)\right| \leq C A_{1} n^{2} .
$$

Now we are ready to estimate the derivatives of $\psi(\xi, \eta)$ from (164). For $\left(\psi_{1}\right)_{\eta}(\xi, \eta)$ after integration by part we have

$$
\begin{aligned}
\left(\psi_{1}\right)_{\eta}= & -\int_{\xi}^{1 / 2} P_{n}\left(\frac{(\xi-\eta) \xi_{1}+2 \xi \eta}{\xi_{1}(\xi+\eta)}\right) \\
& \times \frac{\partial}{\partial \xi_{1}}\left[\frac{\xi_{1}\left(\xi-\xi_{1}\right)}{\eta(\xi+\eta)} \psi^{\prime}\left(\xi_{1}\right)\right] d \xi_{1}+\left(F_{1}\right)_{\eta} \\
& +P_{n}^{\prime}\left(\frac{\xi-\eta+4 \xi \eta}{\xi+\eta}\right) \frac{2 \xi(2 \xi-1)}{(\xi+\eta)^{2}} \psi\left(\frac{1}{2}\right) \\
& +P_{n}\left(\frac{\xi-\eta+4 \xi \eta}{\xi+\eta}\right) \frac{2 \xi-1}{4 \eta(\xi+\eta)} \psi^{\prime}\left(\frac{1}{2}\right) .
\end{aligned}
$$

Then, applying (174), it follows that

$$
\left|\left(\psi_{1}\right)_{\eta}\right| \leq C(\xi+\eta)^{-2}\left[A_{1} n^{2}+C A_{3}\right] .
$$

Similarly, for $\left(\psi_{1}\right)_{\xi}$, we find

$$
\begin{aligned}
\left(\psi_{1}\right)_{\xi}= & -\int_{\xi}^{1 / 2} P_{n}\left(\frac{(\xi-\eta) \xi_{1}+2 \xi \eta}{\xi_{1}(\xi+\eta)}\right) \\
& \times \frac{\partial}{\partial \xi_{1}}\left[\frac{\xi_{1}\left(\eta+\xi_{1}\right)}{\xi(\xi+\eta)} \psi^{\prime}\left(\xi_{1}\right)\right] d \xi_{1}+\left(F_{1}\right)_{\xi} \\
& +P_{n}^{\prime}\left(\frac{\xi-\eta+4 \xi \eta}{\xi+\eta}\right) \frac{2 \eta(2 \eta+1)}{\xi+\eta} \psi\left(\frac{1}{2}\right) \\
& +P_{n}\left(\frac{\xi-\eta+4 \xi \eta}{\xi+\eta}\right) \frac{2 \eta+1}{4 \xi(\xi+\eta)} \psi^{\prime}\left(\frac{1}{2}\right),
\end{aligned}
$$


and thus we get

$$
\left|\left(\psi_{1}\right)_{\xi}\right| \leq C(\xi+\eta)^{-2}\left(A_{1} n^{2}+C A_{3}\right) .
$$

Finally, to prove (102), notice that

$$
\begin{aligned}
\left(F^{n}\right)_{r}(r, t)= & -\left.r^{-2} \psi_{1}(\xi, \eta)\right|_{\substack{\xi=(r+t) / 2 ; \\
\eta=(r-t) / 2}} \\
& +\left.\frac{1}{2} r^{-1}\left(\left(\psi_{1}\right)_{\xi}+\left(\psi_{1}\right)_{\eta}\right)\right|_{\substack{\xi=(r+t) / 2 ; \\
\eta=(r-t) / 2}}, \\
\left(F^{n}\right)_{t}(r, t)= & \left.\frac{1}{2} r^{-1}\left(\left(\psi_{1}\right)_{\xi}-\left(\psi_{1}\right)_{\eta}\right)\right|_{\substack{\xi=(r+t) / 2 ; \\
\eta=(r-t) / 2}},
\end{aligned}
$$

and therefore

$$
\left|\left(F^{n}\right)_{r}(r, t)\right|+\left|\left(F^{n}\right)_{t}(r, t)\right| \leq C r^{-3}\left(A_{1} n^{2}+C A_{3}\right),
$$

where the constant $C$ is independent of $n$ and $f$.

The estimate (101) is a straightforward consequence of formulas (172) for $G^{\prime}$ and (164) for $\psi_{1}$, while the case $n=0$ follows directly from the representation (153).

\section{Conflict of Interests}

The authors declare that there is no conflict of interests regarding the publication of this paper.

\section{Acknowledgments}

The research of Nedyu Popivanov and Todor Popov was partially supported by the Bulgarian NSF under Grant DCVP 02/1/2009 and by Sofia University Grant 94/2014. The research of Allen Tesdall was partially supported by Research Foundation of CUNY Grant 66237-00 44. The authors would like to thank the anonymous referees for the constructive comments and suggestions to improve the quality of the paper.

\section{References}

[1] M. H. Protter, "New boundary value problems for the wave equation and equations of mixed type," Journal of Rational Mechanics and Analysis, vol. 3, pp. 435-446, 1954.

[2] K.-C. Tong, "On a boundary value problem for the wave equation," Science Record, New Series, vol. 1, pp. 1-3, 1957.

[3] N. I. Popivanov and M. Schneider, "The Darboux problems in $\mathbf{R}^{3}$ for a class of degenerating hyperbolic equations," Journal of Mathematical Analysis and Applications, vol. 175, no. 2, pp. 537$578,1993$.

[4] K. Ch. Khe, "On nontrivial solutions of some homogeneous boundary value problems for the multidimensional hyperbolic Euler-Poisson-Darboux equation in an unbounded domain," Differential Equation, vol. 34, no. 1, pp. 139-142, 1998.

[5] N. I. Popivanov and M. Schneider, "On M. H. Protter problems for the wave equation in $R^{3}$," Journal of Mathematical Analysis and Applications, vol. 194, no. 1, pp. 50-77, 1995.

[6] C. S. Morawetz, "A weak solution for a system of equations of elliptic-hyperbolic type," Communications on Pure and Applied Mathematics, vol. 11, pp. 315-331, 1958.
[7] P. D. Lax and R. S. Phillips, "Local boundary conditions for dissipative symmetric linear differential operators," Communications on Pure and Applied Mathematics, vol. 13, pp. 427-455, 1960.

[8] C. S. Morawetz, "Mixed equations and transonic flow," Journal of Hyperbolic Differential Equations, vol. 1, no. 1, pp. 1-26, 2004.

[9] M. N. Jones, Spherical Harmonics and Tensors for Classical Field Theory, vol. 2 of Electronic \& Electrical Engineering Research Studies: Applied and Engineering Mathematics Series, Research Studies Press, Letchworth, UK, 1986.

[10] N. Popivanov and T. Popov, "Singular solutions of Protter's problem for the 3+1-D wave equation," Integral Transforms and Special Functions, vol. 15, no. 1, pp. 73-91, 2004.

[11] V. Müller, Spectral Theory of Linear Operators and Spectral Systems in Banach Algebras, vol. 139 of Operator Theory: Advances and Applications, Birkhäuser, Basel, Switzerland, 2nd edition, 2007.

[12] N. Popivanov, T. Popov, and A. Tesdall, "Semi-Fredholm solvability and asymptotic expansions of singular solutions for Protter problems," in Proceedings of the AIP Conference Proceedings, vol. 1570, pp. 335-342, 2013.

[13] L. Dechevski, N. Popivanov, and T. Popov, "Exact asymptotic expansion of singular solutions for the $(2+1)$-D Protter problem," Abstract and Applied Analysis, vol. 2012, Article ID 278542, 33 pages, 2012.

[14] N. Popivanov and T. Popov, "Exact behavior of singularities of Protter's problem for the 3-D wave equation," in Inclusion Methods for Nonlinear Problems with Applications in Engineering, Economics and Physics, J. Herzberger, Ed., vol. 16 of Computing Supplement, pp. 213-236, 2003.

[15] N. Popivanov, T. Popov, and R. Scherer, "Asymptotic expansions of singular solutions for $(3+1)$-D Protter problems," Journal of Mathematical Analysis and Applications, vol.331, no. 2, pp.10931112, 2007.

[16] N. Popivanov, T. Popov, and R. Scherer, "Protter-Moravetz multidimensional problems," Proceedings of the Steklov Institute of Mathematics, vol. 278, no. 1, pp. 179-198, 2012, International Conference on Differential Equations and Dinamical Systems.

[17] S. A. Aldashev, "Spectral Darboux-Protter problems for a class of multidimensional hyperbolic equations," Ukrainian Mathematical Journal, vol. 55, no. 1, pp. 126-135, 2003.

[18] D. E. Edmunds and N. I. Popivanov, "A nonlocal regularization of some over-determined boundary-value problems. I," SIAM Journal on Mathematical Analysis, vol. 29, no. 1, pp. 85-105, 1998.

[19] J. D. Jeon, K. C. Khe, J. H. Park, Y. H. Jeon, and J. B. Choi, "Protter's conjugate boundary value problems for the twodimensional wave equation," Journal of the Korean Mathematical Society, vol. 33, no. 4, pp. 857-863, 1996.

[20] Ar. B. Bazarbekov and Ak. B. Bazarbekov, "The Goursat and the Darboux problems for the three-dimensional wave equation," Differential Equation, vol. 38, no. 5, pp. 695-701, 2002.

[21] A. V. Bitsadze, Some Classes of Partial Differential Equations, vol. 4 of Advanced Studies in Contemporary Mathematics, Gordon and Breach Science, New York, NY, USA, 1988.

[22] S. Kharibegashvili, "On the solvability of a spatial problem of Darboux type for the wave equation," Georgian Mathematical Journal, vol. 2, no. 4, pp. 385-394, 1995.

[23] S. Kharibegashvili and B. Midodashvili, "On the solvability of one boundary value problem for one class of semilinear second order hyperbolic systems," Journal of Mathematical Analysis and Applications, vol. 400, no. 2, pp. 345-362, 2013. 
[24] S. A. Aldashev, "On the well-posedness of multidimensional Darboux problems for the wave equation," Ukrainian Mathematical Journal, vol. 45, no. 9, pp. 1456-1464, 1993.

[25] J. B. Choi and J. Y. Park, "On the conjugate Darboux-Protter problems for the two dimensional wave equations in the special case," Journal of the Korean Mathematical Society, vol. 39, no. 5, pp. 681-692, 2002.

[26] M. K. Grammatikopoulos, N. I. Popivanov, and T. P. Popov, "New singular solutions of Protter's problem for the 3D wave equation," Abstract and Applied Analysis, vol. 2004, no. 4, pp. 315-335, 2004.

[27] M. K. Grammatikopoulos, T. D. Hristov, and N. I. Popivanov, "Singular solutions to Protter's problem for the 3-D wave equation involving lower order terms," Electronic Journal of Differential Equations, vol. 2003, no. 03, pp. 1-31, 2003, http://ejde.math.txstate.edu/Volumes/2003/03.

[28] A. K. Aziz and M. Schneider, "Frankl-Morawetz problem in $\mathbf{R}^{3}$," SIAM Journal on Mathematical Analysis, vol. 10, no. 5, pp. 913921, 1979.

[29] D. Lupo, K. R. Payne, and N. I. Popivanov, "Nonexistence of nontrivial solutions for supercritical equations of mixed elliptichyperbolic type," in Contributions to Nonlinear Analysis, vol. 66 of Progress in Non-Linear Differential Equations and Their Applications, pp. 371-390, Birkhäuser, Basel, Switzerland, 2006.

[30] P. R. Garabedian, "Partial differential equations with more than two independent variables in the complex domain," Journal of Mathematics and Mechanics, vol. 9, pp. 241-271, 1960.

[31] L. Hörmander, The Analysis of Linear Partial Differential Operators. III, vol. 274, Springer, New York, NY, USA, 1985.

[32] A. Erdélyi, W. Magnus, F. Oberhettinger, and F. G. Tricomi, Higher Transcendental Functions, vol. 1, McGraw-Hill, New York, NY, USA, 1953.

[33] G. Lohöfer, "Inequalities for the associated Legendre functions," Journal of Approximation Theory, vol. 95, no. 2, pp. 178-193, 1998.

[34] E. M. Stein, Singular Integrals and Differentiability Properties of Functions, vol. 14 of Princeton Mathematical Series, No. 30, Princeton University Press, Princeton, NJ, USA, 1970.

[35] E. T. Copson, "On the Riemann-Green function," Archive for Rational Mechanics and Analysis, vol. 1, pp. 324-348, 1958.

[36] S. G. Samko, A. A. Kilbas, and O. I. Marichev, Fractional Integrals and Derivatives: Theory and Applications, Gordon and Breach Science, Yverdon, Switzerland, 1993. 


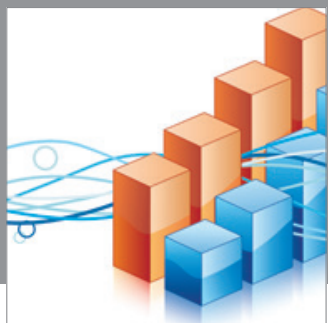

Advances in

Operations Research

mansans

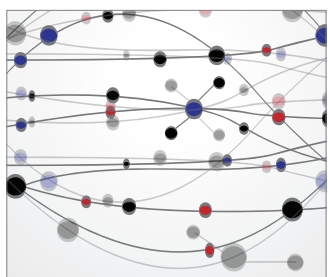

The Scientific World Journal
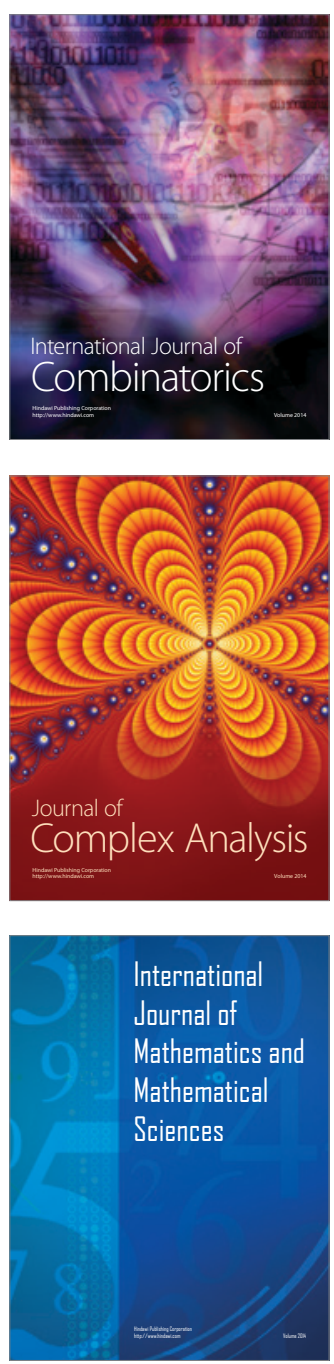
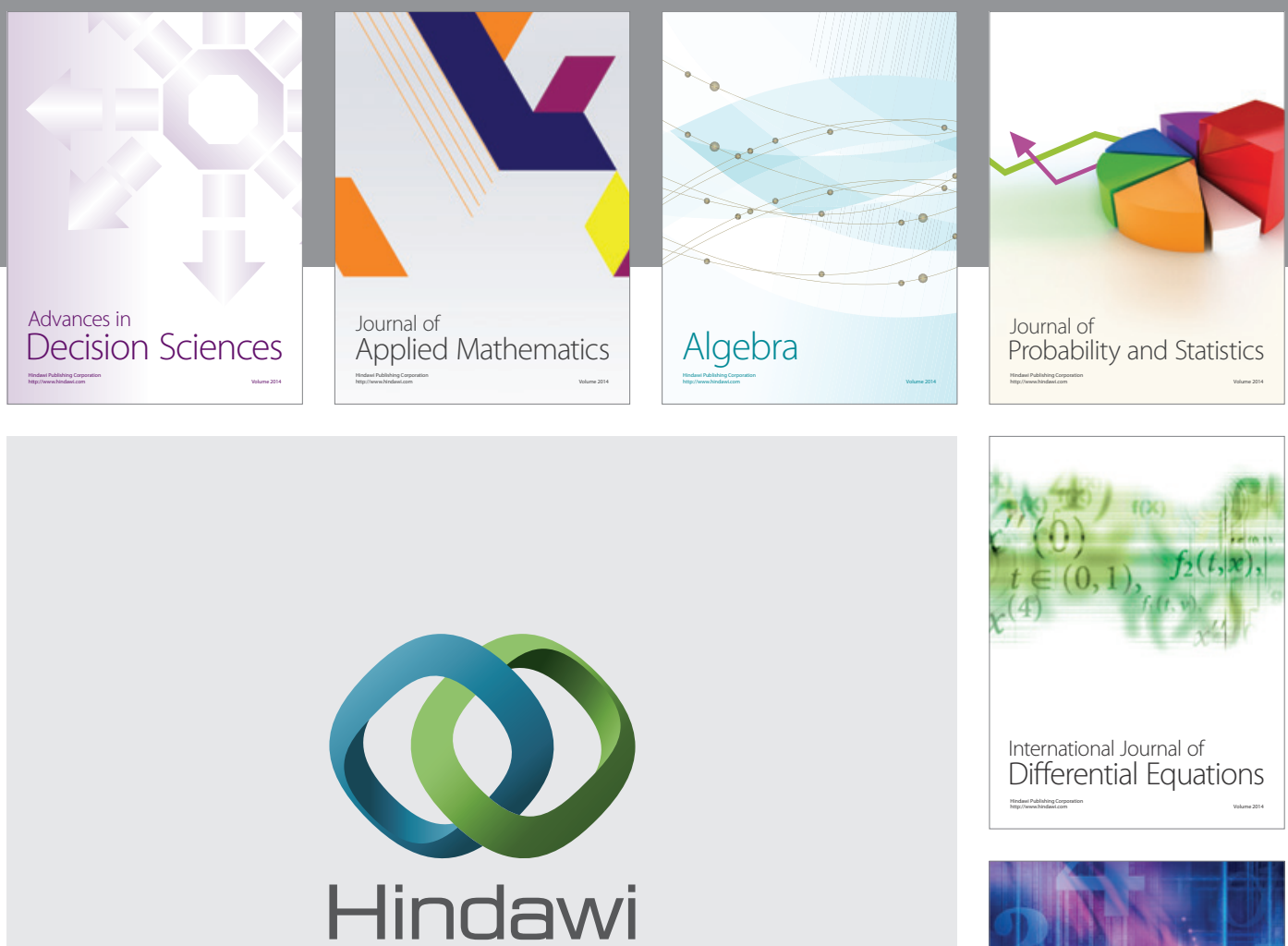

Submit your manuscripts at http://www.hindawi.com
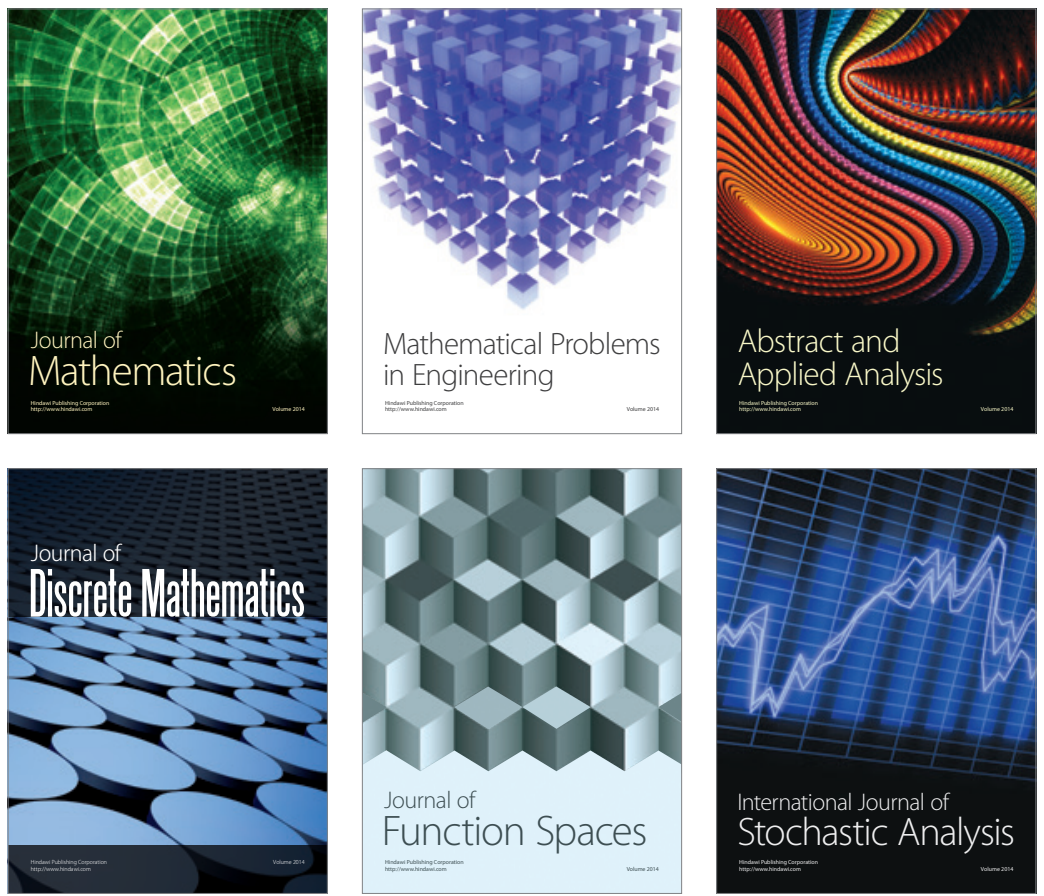

Journal of

Function Spaces

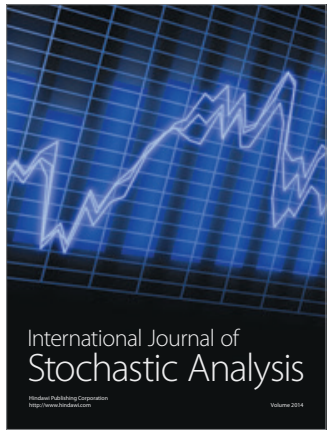

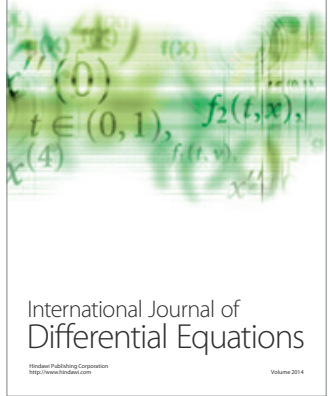
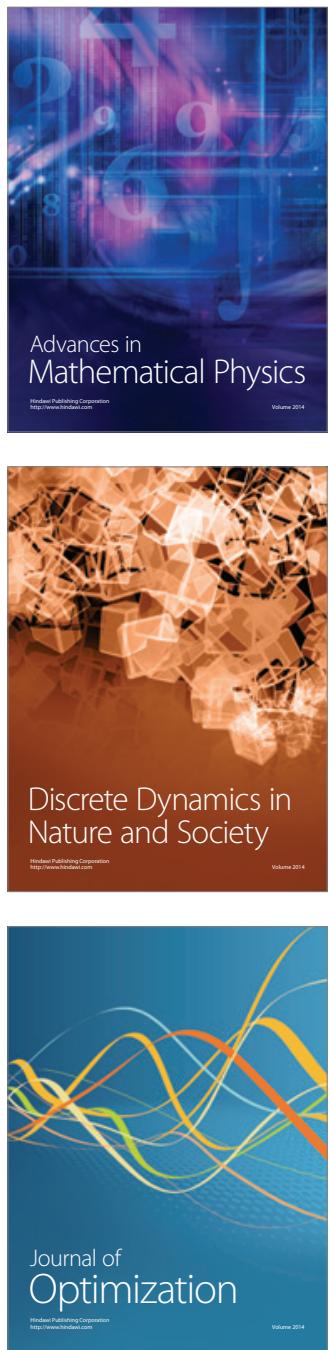